
Physical Chemistry B 124( 39) : 8465-8478 (2020) copyright () 2020 American Chemical Society after peer review and technical editing by the publisher. To access the final edited and published work see https://doi.org/10.1021/ acs.jpcb.0c04769

\title{
Emerging Ionic Soft Materials based on Deep Eutectic Solvents
}

\author{
Liliana C. Tomé ${ }^{*}$ and David Mecerreyes ${ }^{a\lrcorner *}$
}

POLYMAT, University of the Basque Country UPV/EHU, Joxe Mari Korta Center, Avda. Tolosa 72, 20018 Donostia-San Sebastian, Spain

'IKERBASQUE, Basque Foundation for Science, 48013 Bilbao, Spain

\section{ABSTRACT}


In the last five years, the use of deep eutectic solvents (DESs) have been opening new perspectives towards the creation of novel ionic soft materials as alternatives to expensive ionic liquids. This Mini-Review highlights the progress and advances in soft ionic materials or gels, mostly composed by a DES immobilized within difference matrices, such as linear polymers, polymer networks, biopolymers, supramolecular compounds or sol-gel derived silica networks. By taking advantage of the DESs characteristics and properties in the solid state, this building system delivers a variety of tailor-made materials showing different functionalities (ionic conductivity, self-healing, stretchability and $\mathrm{pH}$-responsiveness) and offers a way to circumvent drawbacks related to shaping and risk of leakage in many technological applications. In this context, we provide a judicious analysis of these emerging ionic soft materials, their properties and applications open in energy, (bio)electronics, drug delivery, analytical chemistry, and wastewater treatment. Perspectives and opportunities for future research directions on this blossoming field are also discussed.

\section{KEYWORDS}

Deep Eutectic Solvents; Soft-materials; Gels; Eutectogels; Ionic Conductivity; Batteries; Supercapacitors; Sensors; Drug delivery.

\section{INTRODUCTION}


Ionic soft materials such as gels or colloids are important in a wide range of technological applications. Gels are semi-solids that can have properties ranging from soft and weak to hard and tough. Gels are usually a bicomponent system where the liquid is the predominant component and the second component acts as gelator forming a three dimensional network. Depending on the liquid component used to form the gel, they can be classified as hydrogels, when the liquid phase is water, or organogels when the liquid phase is an organic solvent. More recently, ionic liquid gels known also as ionogels or iongels were proposed as an additional type of gels. Iongels have some similarities to hydrogels since they are ionic in nature, and to organogels due to its organic nature. The ionic liquid is usually the main component and an additional second component, such as a polymer, nanoparticle or sol-gel derived silica network, acts as gelator forming a threedimensional network. Since their discovery, ${ }^{1-3}$ iongels have become a very popular type of ionic soft materials finding different applications in energy, ${ }^{4}$ (bio)electronics, ${ }^{5}$ or environmental science. $^{6}$

Deep Eutectic Solvents (DESs) are a relatively new class of solvents that are often considered to be a sub-class of ionic liquids having similar properties, including ionic nature, tunability, low vapour pressure, low flammability, and relatively wide liquid range. ${ }^{7}$ Additionally, DESs benefit from remarkable advantages over ionic liquids, such as the low cost of components, high biobased content and its easy fabrication. ${ }^{8,9}$ Altogether, these advantages place DESs in the forefront of ionic liquid's applicability within a sustainable framework. Therefore, the number of applications of DESs in materials science has been growing constantly during the last decade, particularly in polymer science and engineering, ${ }^{10-12}$ metal processing, ${ }^{7}$ and nanomaterials. ${ }^{13}$

Although DESs were soon identified as an essential tool in the preparation of innovative materials with enhanced functionalities, the application of DESs in the preparation of ionic soft 
materials is a relatively recent research topic. Only in the last 5 years, the use of DESs is becoming popular due to its sustainable and economically viable approach as alternatives to expensive ionic liquids. In this Mini-Review, we aim to highlight and critically analyse the progress and advances on the development of ionic soft materials or gels based on DESs (Figure 1). We first introduce the different DESs, types of gelators, physico-chemical properties and combinations explored so far. Then, we focuses on their applications open in the fields of energy, (bio)electronics, drug delivery, analytical chemistry, and wastewater treatment, with the intent of consolidate current achievements and promote further developments. Perspectives and opportunities for future research directions are finally discussed, particularly regarding the importance of providing innovative materials not only to enhance performance, but also to deliver a cost-effective and sustainable solution.

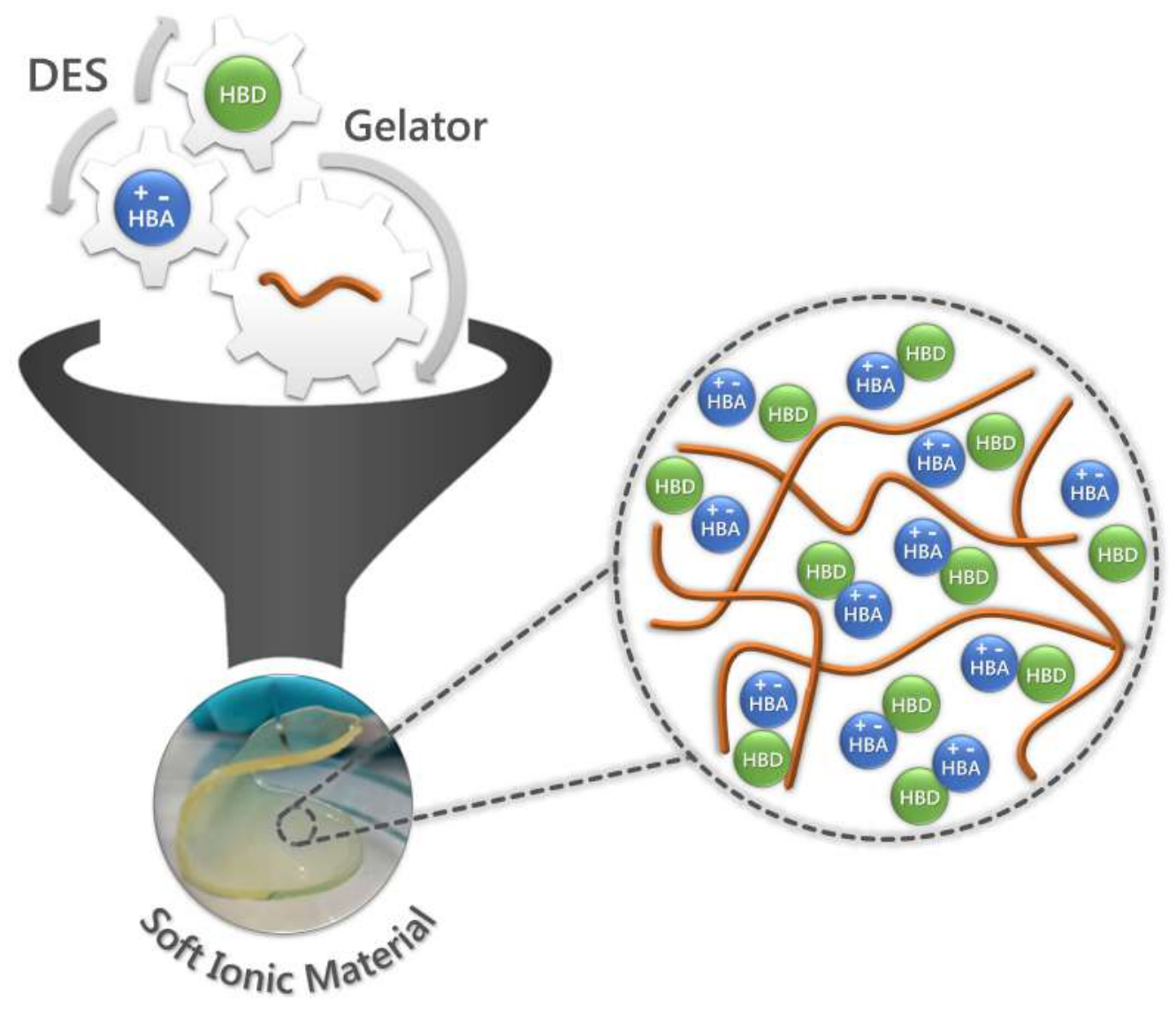


Figure 1. Schematic representation of an ionic soft material, in which the DES is the predominant component and the second component acts as gelator forming a three dimensional network

\section{TOWARDS IONIC SOFT MATERIALS BASED ON DESs}

\subsection{Deep Eutectic Solvents (DESs)}

The term "deep eutectic solvent" (DES) was first introduced in 2003 by Abbott, ${ }^{14}$ who defined a DES as a liquid mixture of compounds having a melting point significantly lower compared to the starting materials. These liquid mixtures were termed deep eutectic solvents to differentiate them from ionic liquids, which are single compounds. DESs are usually obtained by complexation of a hydrogen bond acceptor (HBA) with a hydrogen bond donor (HBD). The charge delocalization occurring through hydrogen bonding between for example a halide ion and the HBD generates a larger nonsymmetrical ion, which have low lattice energy and hence low melting points.?

Numerous combinations of different families of components that show strong intermolecular hydrogen bonding interactions have been reported to form DESs. However, the most popular involve HBAs like quaternary ammonium salts, especially choline chloride $(\mathrm{ChCl})$, and $\mathrm{HBDs}$ selected from a wide range of natural compounds, such as organic acids, alcohols or sugars. ${ }^{15}$ Compared to ionic liquids, these type of DESs are less toxic and less expensive, and similarly, the ability of tailor-made these solvents with the most adequate properties for a given application is still enormous.$^{16}$ Moreover, their synthesis is straightforward and fully embrace the principles of Green Chemistry, ${ }^{17}$ as it consists of simply mixing HBDs and HBAs without any reaction, purification step, or generation of waste.

Several excellent reviews describing the synthesis, properties and applications of DESs have been published in the last few years. ${ }^{7,15,16,18-20}$ For this reason, we will not discuss in detail the DES component, but we will highlight the different DESs used in the design of ionic soft materials. Figure 2 shows some examples of the chemical structures of DES components investigated so far. Most of the ionic soft materials have been designed using DESs prepared with readily available, nontoxic precursors, specifically ChCl-based DESs involving various HBDs 
such as urea, glycerol, polyols, sugars or organic acids. It should be mentioned that mixtures of $\mathrm{ChCl}$ and carboxylic acids are one of the most commonly used DESs, but this family was found to degrade due to an esterification reaction between the carboxylic acid and the alcohol moiety of $\mathrm{ChCl}^{21}$ Although the addition of water can avoid or slow down the esterification, this side reaction occurs even at room temperature over extended periods of time and is promoted at elevated temperatures. Therefore, the applicability of $\mathrm{DESs}$ based on $\mathrm{ChCl}$ and carboxylic acids can be compromised.

Overall, the biobased and sustainable nature of DESs has driven the interest as the basic component of ionic soft materials. However, there are other types of toxic and hazard DESs that can be prepared by mixing fluorinated lithium salts (i.e. LiTFSI) and HBDs like urea and Nmethylacetamide, which have shown specific technological interest, as it will be discussed later.

It is also worth to mention here the development of DESs that can be directly polymerized leading to ionic polymers. Thus, Del Monte and Luna-Bárcenas, reported a series of polymers from the polymerization of DESs based on mixtures of $\mathrm{ChCl}$ and acrylic acid as HBD. These DES monomers showed to be suitable for frontal polymerization methods,,$^{22,23}$ and the elaboration of carbon nanotube (CNT) nanocomposites. ${ }^{24}$ Using the DES monomer strategy, Mecerreyes' group designed a cholinium methacrylate bromide salt (HBA monomer), ${ }^{25}$ which formed DESs with a variety of HBDs such as citric acid, maleic acid or diamines. ${ }^{26}$ By conventional free radical polymerization a series of new ionic polymers alternative to poly(ionic liquid)s were reported showing interesting properties as $\mathrm{CO}_{2}$ sorbents. 


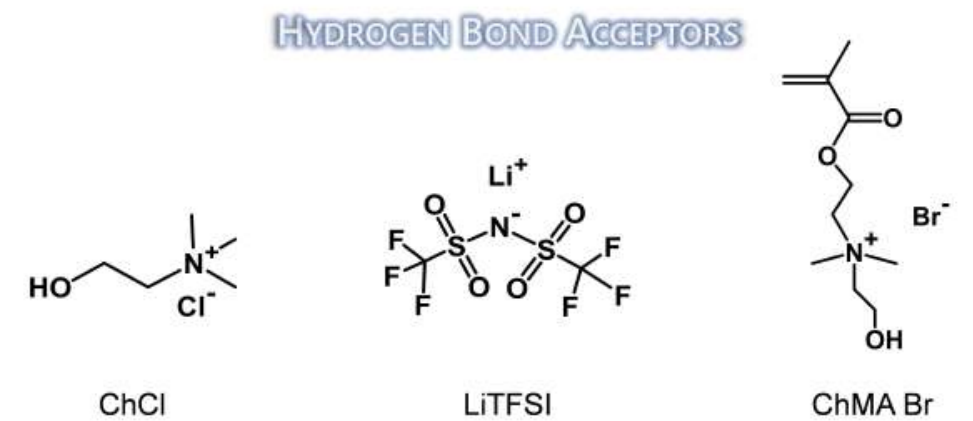

\section{HYYROGEN BOND DONORS}

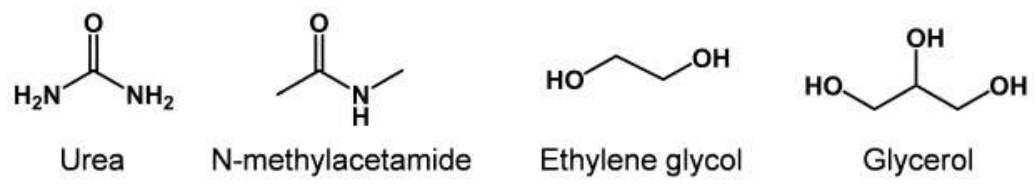

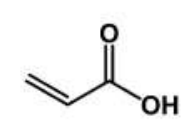

Acrylic acid<smiles>O=C(O)CC(O)(CC(=O)O)C(=O)O</smiles>

Citric acid<smiles>Cc1cc(O)cc(O)c1</smiles>

Orcinol<smiles>O=C(O)Cc1ccccc1</smiles>

Phenylacetic acid<smiles>OC[C@@H](O)[C@@H](O)[C@H](O)[C@H](O)CO</smiles>

Sorbitol<smiles>O=C1O[C@H]([C@@H](O)CO)C(O)=C1O</smiles>

Ascorbic acid<smiles>O=C(O)C(=O)O</smiles>

Oxalic acid<smiles>O=C[C@H](O)[C@@H](O)[C@H](O)[C@H](O)CO</smiles>

Galactose<smiles>O=C(CO)[C@@H](O)[C@H](O)[C@H](O)CO</smiles>

Fructose

Figure 2. Examples of chemical structures of hydrogen bond acceptors (HBAs) and donors (HBDs), which have been combined in DESs and used to create diverse ionic soft materials.

\subsection{Types of Gelators}

The second component of gel materials is the gelator, which forms the three-dimensional network within the DES and it is the responsible for the solid-like behavior. Different types of gelators have been used, such as linear polymers, polymer networks, biopolymers, supramolecular compounds or sol-gel derived silica networks (Figure 3). 


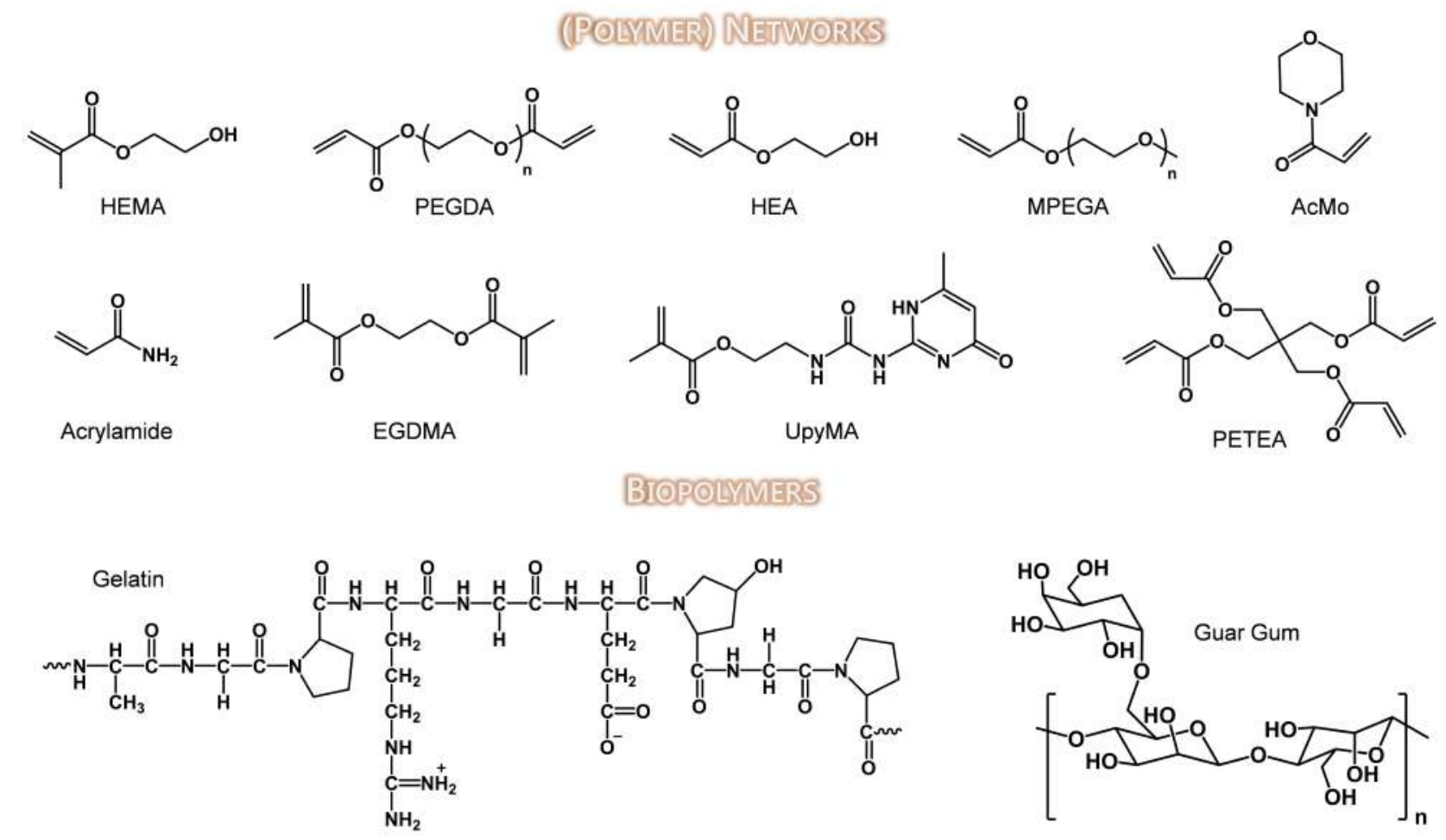

LOW MUCLEGUAR WEGHT GELATORS

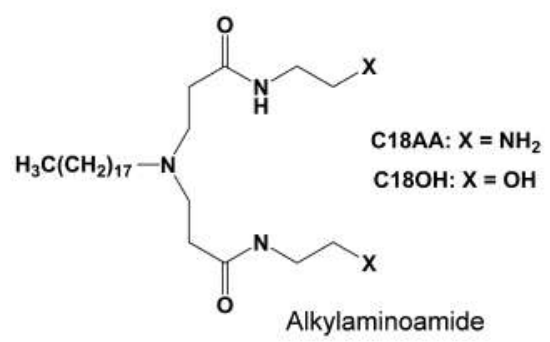

SOL-GER DERTVED STICA NETWORKS

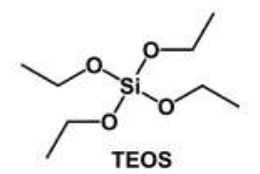

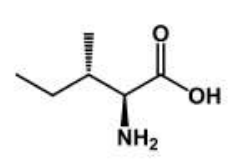

Isoleucine

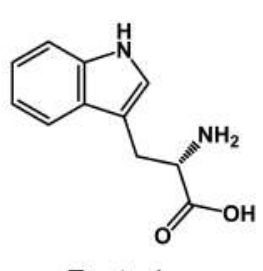

Tryptophan

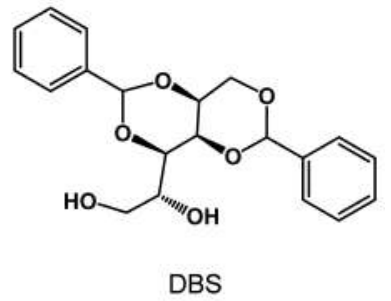

INNORGANTE NANOPARUICLES

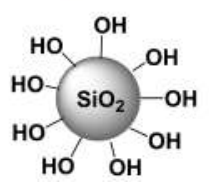

Figure 3. Chemical structures of the different gelators used to form ionic soft materials.

The incorporation of non-polymerizable DESs within acrylic polymer matrices is perhaps the most straightforward strategy to obtain ionic soft materials based on DESs. In 2016, Mukesh et al. ${ }^{27}$ investigated the self-polymerization ability of three monomers namely 2-hydroxyethyl methacrylate (HEMA), N-isopropylacrylamide (NIPAM) and vinyl acetate (VA) in $\mathrm{ChCl} / \mathrm{Orcinol}$ 
(1:1.5), without adding any initiator. Amongst the three monomers, only HEMA was found to selfpolymerized at room temperature and optimized concentrations. This group also reported on the self-polymerization of HEMA in the presence of $\mathrm{ChCl} /$ Fructose $(2: 1)$ and an anti-inflammatory drug ${ }_{.28}$ Along this line, Mokhtarpour et al..$^{29}$ have recently developed stable ionic soft materials containing an anti-cancer drug, $\mathrm{ChCl} /$ ascorbic acid (2:1) and HEMA. The authors studied the effect of $\mathrm{pH}$ on self-polymerization of HEMA and found that best $\mathrm{pH}$ range for gel formation is between $\mathrm{pH} 1.7$ and 3.5 .

Conventional thermal free radical polymerization is the most popular chemical pathway for the development of ionic soft materials based on DESs. For instance, Hong et al.$^{30}$ fabricated ionic soft materials by in situ free radical polymerization of acrylamide in the presence of $\mathrm{ChCl} /$ Urea/Glycerol (1:2:0.125), a small amount of the cross-linker $N, N$ '-methylenebisacrylamide, and cellulose pulp as a reinforcement material. In this case, the DES is locked in the PAAM polymer matrix through noncovalent bonding (hydrogen bond and van der Waals' interactions). Very recently, Jaumaux et al. ${ }^{31}$ reported a DES gel material based on a copolymer matrix, which was synthesized in situ by thermally polymerizing 2-(3-(6-methyl-4-oxo-1,4-dihydropyrimidin-2yl)ureido) ethyl methacrylate (UPyMA) and pentaerythritol tetraacrylate (PETEA) monomers in the presence of LiTFSI/N-methylacetamide (1:4) and fluoroethylene carbonate (FEC) as additive.

Alternatively, Logan et al..$^{32}$ used a more facile and rapid synthetic route using UV-initiated free radical polymerization of an acrylate monomer mixture. Stable and robust ionic soft materials with $70 \mathrm{wt} \%$ LiTFSI/N-methylacetamide (1:4) were prepared using different rations of poly(ethylene glycol) methyl ether acrylate (MPEGA) and 2-hydroxyethyl acrylate (HEA) monomer, while keeping the cross-linker poly(ethylene glycol) diacrylate (PEGDA) at the absolute minimum required to obtain free-standing materials. ${ }^{32}$ Actually, the use of UV- photopolymerized polymer networks is a worthy option to fabricate ionic soft materials with high DES content and low polymer content. Qin and Panzer, ${ }^{33}$ incorporated $\mathrm{ChCl} /$ Ethylene glycol (1:2) into chemically crosslinked polymer scaffolds (ranging from 13.2 to 38.4 vol\% polymer content) formed via UV curing of HEMA and PEGDA. Note that HEMA or HEA monomers have been commonly used not only 
due to the reactive nature of acrylate and methacrylate in free radical polymerization, but also because to its $-\mathrm{OH}$ functional group, which is supposed to facilitate a good miscibility with DESs. On the other hand, Joos et al. ${ }^{34}$ prepared UV-cured ionic soft materials combining LiTFSI/Nmethylacetamide (1:4) ranging from 65 to 90 vol\% content, and a polymer network with varying molar compositions of acryloylmorpholine (AcMo) and ethylene glycol dimethacrylate (EGDMA). In this case, the AcMo monomer was chosen to maximize its miscibility with the polar DES and prevent possible phase separation. Nevertheless, one of the main challenges regarding the use of polymers as gelators is to avoid the leaching of DES from the gel material due to the miscibility problems between the polymer and IL components.

Another type of gelators commonly used are biopolymers. The use of biopolymers offers an appealing way to advance gel biocompatibility and exploit self-assembly strategies (e.g. Hbonding) designed by nature. Mukesh and Prasad reported for the first time the preparation of three different structural formats of DNA in a single DES, specifically $\mathrm{ChCl} /$ Ethylene glycol (1:2). ${ }^{35}$ Panzer and coworkers presented a new class of gelatin-based gels featuring $\mathrm{ChCl} /$ Ethylene glycol (1:2) ${ }^{36}$ These ionic soft materials were synthesized in a facile manner using the thermally modulated coil-helix transition process of gelatin induced by heating and subsequently cooling the gelatin/DES mixture. In addition, Depoorter et al..$^{3 r}$ developed fully biosourced gel materials synergizing the attributes of guar gum polysaccharide and a series of DESs involving choline chloride and various HBDs, such as urea, di or triacids, polyols or carbohydrates. The polar character of these HBDs was expected to exalt the chemical affinities with guar chains. However, Guar-ChCl/glycerol and Guar-ChCl/oxalic acid mixtures exhibited a clear macroscopic de-mixing, while the other combinations lead to optically homogeneous mixtures. The Guar-ChCl/urea formed the most promising gels, displaying a solid-like behavior, which resulted from the synergistic $\mathrm{H}$-bonding between the $-\mathrm{OH}$ groups of guar gum and the multiple sites of complexation offered by $\mathrm{ChCl} / \mathrm{urea}\left(-\mathrm{OH},-\mathrm{Cl} \text {, and }-\mathrm{NH}_{2}\right)^{37}$

Despite that one of the most popular type of gelators under the umbrella of iongels are sol-gel derived silica networks ${ }^{38}$ there is only one work reporting on DESs as alternative dispersed liquid 
phases. Joos et al., ${ }^{39}$ who proposed the name of "eutectogels", introduced a new class of ionic soft materials in which a DES is confined within a silica matrix. These ionic soft materials were prepared via a non-aqueous sol-gel route and the DES (i.e. LiTFSI and N-methylacetamide) used in the formulation revealed to be compatible with the acid conditions of the sol-gel process. Conversely, the authors found that the heating step, required to remove the side products from the silica's hydrolysis and condensation, removes indiscriminately a fraction of the $\mathrm{N}$ methylacetamide as well. ${ }^{39}$ The main difficulty here is that the use of sol-gel method and reactants will be strongly dependent on the type of DES.

An additional type of gelator are inorganic nanoparticles. Solid-like soft materials called colloidal gels can be formed in particular cases by using different inorganic nanoparticles and DESs. For example, a transparent ionic soft material can be obtained by dispersing $10 \mathrm{wt} \% \mathrm{SiO}_{2}$ nanoparticles (Aerosils 320) into $\mathrm{ChCl} /$ Ethylene glycol (1:2), due to hydrogen bonding and possible electrostatic or Van der Waals interactions between the DES molecules and the silica nanoparticles..$^{40}$ Although similar ionic soft materials can also be obtained by adding another kind of hydrophilic aerosils $\left(\mathrm{SiO}_{2}-200\right)$, gel formation was not observed when hydrophobic aerosils $\left(\mathrm{SiO}_{2}-812\right.$ and $\left.\mathrm{SiO}_{2}-974\right)$ were added to $\mathrm{ChCl} /$ Ethylene glycol $(1: 2){ }^{40}$ This suggested that the $-\mathrm{OH}$ groups on the hydrophilic $\mathrm{SiO}_{2}$ surface can possibly form hydrogen bonds with the DES, playing a key role on the dispersion and gelator capability of $\mathrm{SiO}_{2}$ nanoparticles. Furthermore, bucky gels or ionic soft materials formed by mixing magnetic multi-walled carbon nanotubes and DESs have also been fabricated without any additional stabilizer. ${ }^{41,42}$

The formation of supramolecular gels bearing DESs immobilized in networks of self-assembled low-molecular-weight gelators (LMWGs) have been attracting particular attention, mainly due to their intriguing properties and fast preparation. Held together because of reversible intermolecular non-covalent interactions, supramolecular gel materials often display thermal reversibility and/or self-healing ability.43 In 2018, D'Anna and coworkers introduced the first example of supramolecular gels in DESs, ${ }^{4+}$ in which concentrations as low as $3 \mathrm{wt} \%$ of L-amino acids (isoleucine and tryptophan) were used to jellify $\mathrm{ChCl} /$ phenylacetic acid (1:2). Gelation was driven 
by the ability of the L-amino acids to interact with the hydrogen bond network of the DES, resulting in strong and fully natural ionic soft materials. ${ }^{44}$ Afterward, Ruiz-Olles et al. ${ }^{45}$ also found that a commercially available LMWG, 1,3:2,4-Dibenzylidene-d-sorbitol (DBS), self-assemble at loadings of $5 \% \mathrm{w} / \mathrm{v}$ into supramolecular gels in DESs based on $\mathrm{ChCl}$ combined with diverse alcohols (ethylene glycol, glycerol, sorbitol, xylitol) or urea. Interestingly, the results showed that underpinning nanofibrillar DBS networks were formed, while the DES nature of the liquid-phase was retained..$^{45}$ In addition, Delbecq et $a l .{ }^{46}$ prepared supramolecular gels of $\mathrm{ChCl} /$ urea (1:2) using two different long alkylaminoamide gelators, bearing two amide groups with two terminal hydroxyl $(\mathrm{C} 18 \mathrm{OH})$ or primary amine moities (C18AA). Overall, the use of LMWGs is a simple, cost-effective, and environmentally friendly way of imparting DESs with desirable ionic soft material properties.

\subsection{Physico-Chemical Properties of Ionic Gels based on DESs}

Interestingly, the gel materials usually preserve the intrinsic properties of the dispersed liquid phase, specifically of the DESs, including thermal stability, low volatility, non-flammability, electrochemical and chemical stability, high ionic conductivity, or good solvation. The properties of these ionic soft materials can be tailored by changing the chemical structure of the DES and gelator, the ratios between them, as well as the nature of interactions (covalent vs non-covalent) and the cross-linking degree. Amongst the different properties of DES gel materials, the conventional ones are ionic conductivity and the mechanical and rheological properties. Furthermore, diverse ionic soft materials have been synthesized offering additional properties or functionalities, such as self-healing, stretchability and $\mathrm{pH}$-responsiveness. In this section, we will discuss key properties and highlight some examples of DES gel materials showing additional functionalities.

The high ionic conductivity is one of the most interesting properties of DES gel materials when being used as electrolytes in electrochemical devices like batteries or supercapacitors. The ionic 
conductivity is proportional to the relative content of the DES to the gelator. Indeed, by increasing the amount of DES, the ionic conductivity of the ionic soft material is usually enhanced, getting very close to the conductivity values of the neat DES. As shown in Figure 4a, this trend was very well illustrated by Joos et al., ${ }^{34}$ who reported a series of ionic soft materials with different volume contents $(65,75,85$ and 95 vol\%) of LiTFSI/N-methylacetamide (1:4) DES in the EGDMA/AcMo precursor (10:90 molar ratio). Nonetheless, an excess of DES can cause poor mechanical stability and phase separation. Particularly in this work, no detrimental effect on the structural integrity was observed up to 85 vol\%, but the sample with 90 vol\% of DES was found to phase separate and the gel material was torn easily. ${ }^{34}$

Temperature $\left({ }^{\circ} \mathrm{C}\right)$
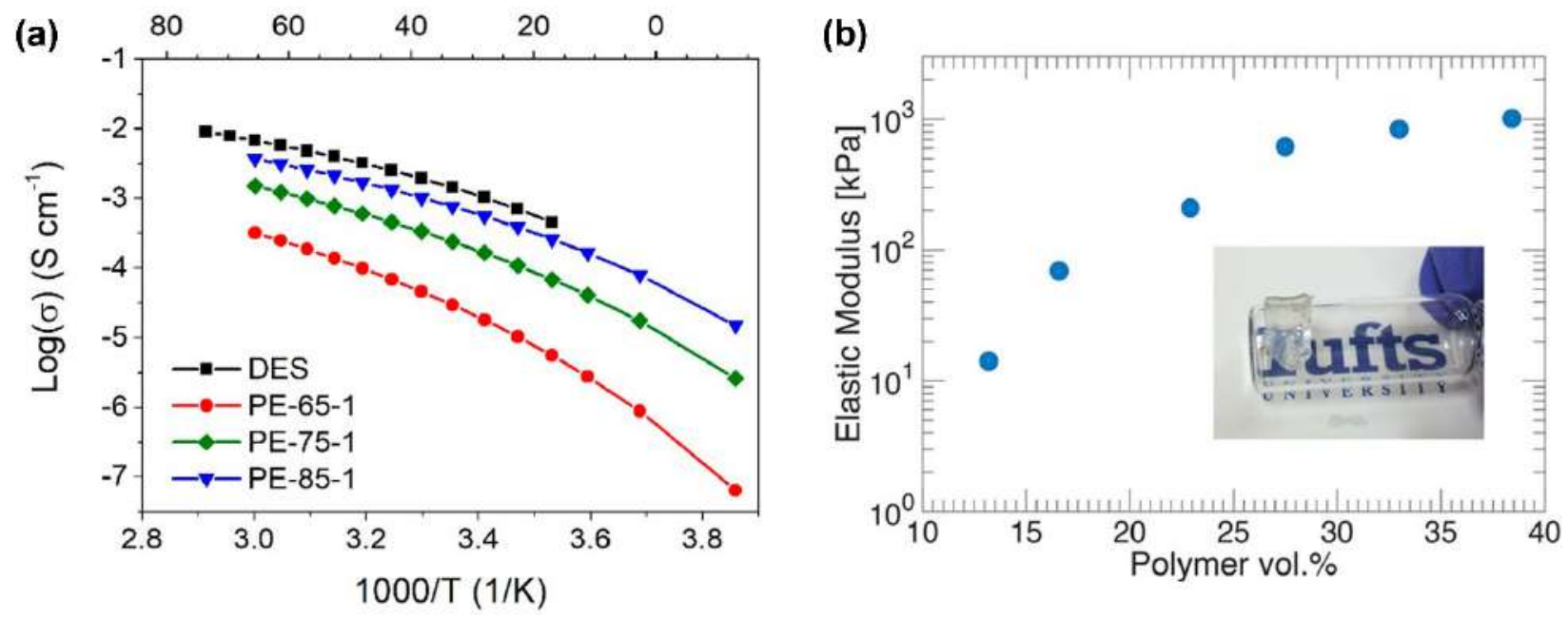

(c)
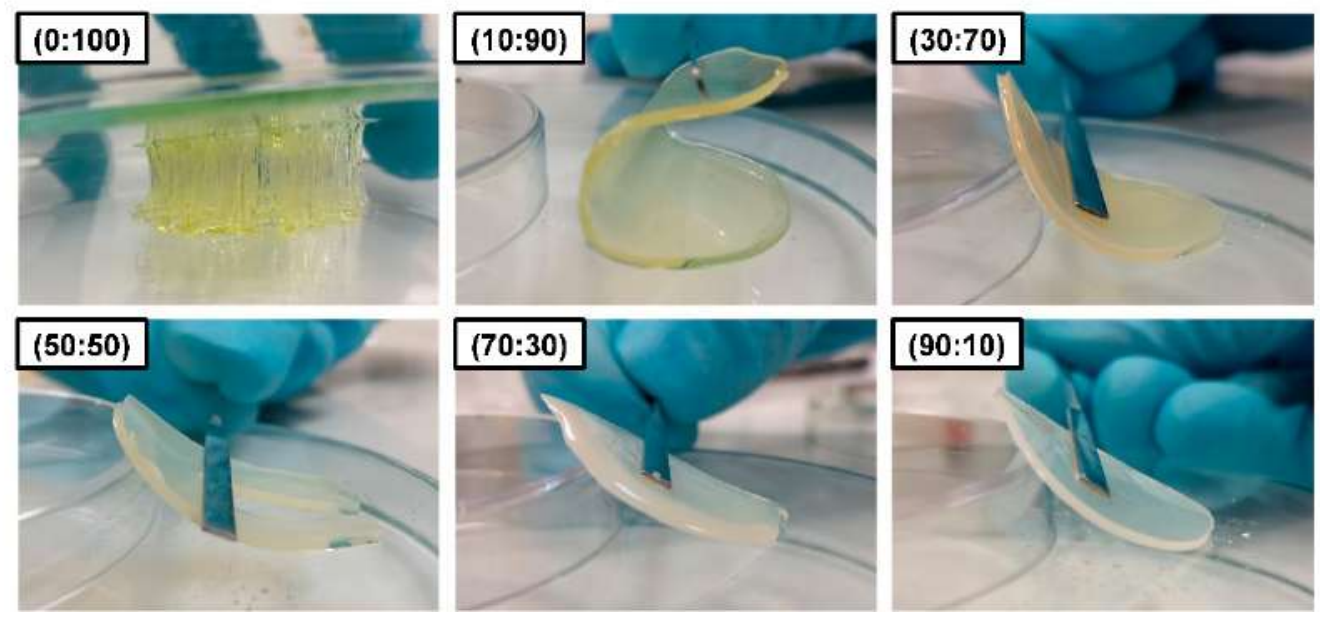
Figure 4. (a) Ionic conductivity of LiTFSI/N-methylacetamide (1:4) (DES) and respective gel material samples (PE-X-Y) containing variable DES volume contents (X-value from 65 to 85 vol\%) and EGDMA/AcMO polymer (10:90 molar ratio, $\mathrm{Y}=1)$. Reprinted with permission from Joos et al ${ }^{34}$ Copyright 2020, American Chemical Society. (b) Compressive elastic modulus of DES gel materials based on $\mathrm{ChCl} /$ Ethylene glycol (1:2) and varying contents of the polymer scaffold HEMA/PEGDA (30:1 molar ratio). Reproduced with permission from Qin and Panzer. ${ }^{33}$ Copyright 2017, John Wiley and Sons. (c) Optical photographs of DES gel materials containing 75 vol\% LiTFSI/N-methylacetamide (1:4) and different molar compositions (x:y) of EGDMA/AcMO polymer. Adapted with permission from Joos et al. Copyright 2020, American Chemical Society.

Logan et al.32 studied the ionic conductivity of ionic soft materials containing 70 wt $\%$ LiTFSI/N-methylacetamide (1:4) and different weight contents of MPEGA and HEA monomers, as well as a minimum amount of PEGDA crosslinker. The authors found that the ionic conductivity decrease with increasing HEA content, probably because the hydroxyl groups of HEA also act as a hydrogen bond accepter and disrupt the DES structure. Ionic conductivities of 0.64 and $1.82 \mathrm{mS} \mathrm{cm}$ at $20{ }^{\circ} \mathrm{C}$ and $40{ }^{\circ} \mathrm{C}$, respectively, were obtained for the gel material made without the HEA monomer. Therefore, considering the ionic conductivity of the liquid DES (1.35 $\mathrm{mS} \mathrm{cm}^{-1}$, the results indicated that the formation of DES gel materials do not dramatically compromise ionic conductivity when compared to that of the liquid alone..$^{32}$

The mechanical and rheological properties of DES gel materials are important factors to take into account according to targeted applications. In general, the chemical nature and ratio between the DES and the gelator are the most relevant parameters affecting the mechanical and rheological properties of these ionic soft materials. As an example, Figure $4 \mathrm{~b}$ shows compressive elastic modulus values obtained for DES gel materials comprising $\mathrm{ChCl} /$ Ethylene glycol (1:2) and varying contents of the polymer scaffold HEMA/PEGDA (30:1). ${ }^{33}$ The elastic modulus significantly increases when increasing the amount of polymer scaffold, due to an increase of polymer entanglement density. In fact, the elastic modulus of the DES-based gel at the minimum gelation point $(14 \mathrm{kPa})$ is comparable to a soft gelatin hydrogel, while the DES-based gel containing $38.4 \mathrm{vol} \%$ polymer scaffold exhibits an elastic modulus of $1.0 \mathrm{MPa}$, which is comparable to a stiff commercial rubber material. ${ }^{33}$ It is noteworthy that the cross-linker is of significant importance to the flexibility of this type of ionic soft materials (covalently cross-linked 
polymers). The increase of cross-linker content can lead to less flexible and very brittle gel materials (Figure 4c).34

Additionally, Depoorter et al. ${ }^{37}$ investigated DES gel materials combining guar gum and found that their viscoelastic properties are closely governed by the hydrogen bond donor species engaged in the ChCl-based DESs. For instance, the rheological behavior of Guar-ChCl/citric acid and Guar- $\mathrm{ChCl} /$ isosorbide totally differs from the one of $\mathrm{Guar}-\mathrm{ChCl} / \mathrm{urea}$, for which an elasticdominated behavior $\left(\mathrm{G}^{\prime}\right.$ higher than $\left.\mathrm{G}^{\prime \prime}\right)$ was observed at high guar gum concentration $(20 \mathrm{wt} \%)$. On the contrary, Guar- $\mathrm{ChCl} /$ citric acid and $\mathrm{Guar}-\mathrm{ChCl} /$ isosorbide behaved as viscous liquids $\left(\mathrm{G}^{\prime \prime}\right.$ superior to $\mathrm{G}^{\prime}$ ), even at high guar gum concentration. The obtained results showed that the rheological behavior of Guar/DES gels depends on the compromise between DES/DES, Guar/Guar (intra- and inter-chain), and Guar/DES interactions. The $\mathrm{ChCl} /$ urea showed the most favorable interactions with guar gum. This can be attributed not only to the lower viscosity of $\mathrm{ChCl} /$ urea that enables a more efficient dissolution of guar gum, but also to the chaotropic nature of urea which acts as an effective hydrogen bond breaker between guar chains $(-\mathrm{C}=\mathrm{O}$ of urea is an HBA site, while $-\mathrm{NH}_{2}$ is an HBD site) ${ }^{37}$

Ionic soft materials combining DESs and exhibiting shape-memory and self-healing properties, allowing for their recovery and repair, have also been developed. Generally, the ability of gel materials to undergo self-healing and shape memory is enhanced by multiple forms of noncovalent supramolecular interactions, as the combination of hydrogen bonding between the DESs and the polymeric gelators. To illustrate with particular examples, Jaumaux et al. ${ }^{31}$ reported an gel material formed from LiTFSI/N-methylacetamide (1:4) and the self-healing UPyMA-PETEA copolymer. This ionic soft material completely self-heal within 2 hours at room temperature, after being cut. Optical images of the self-healing process are shown in Figure 5a. 
(a)

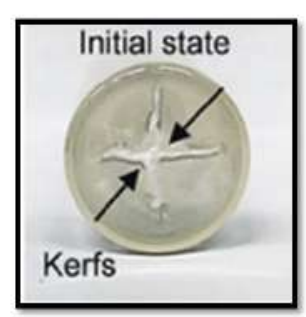

(c)
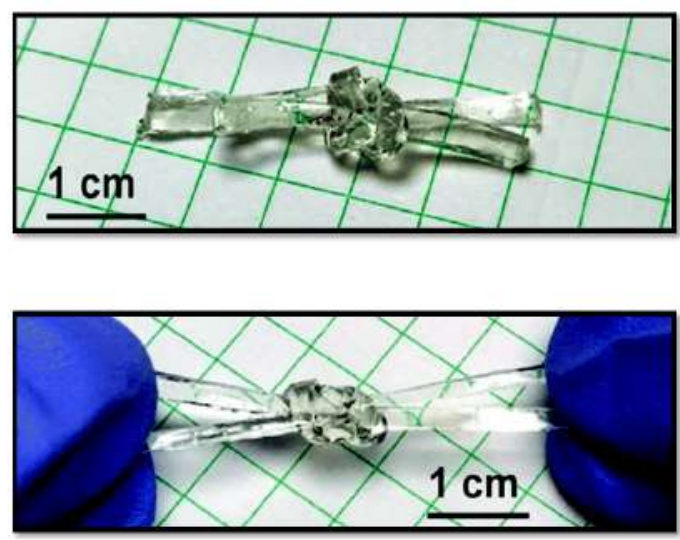

(b)

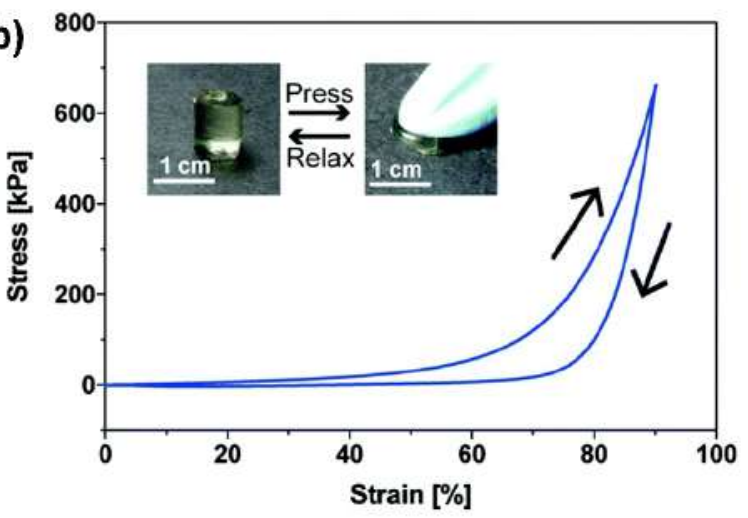

(d)
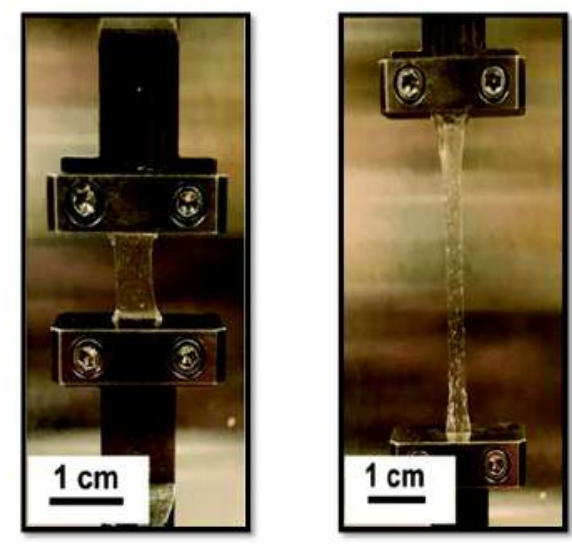

Figure 5. (a) Optical images of the self-healing process of an ionic soft material made of LiTFSI/Nmethylacetamide (1:4) and UPyMA-PETEA copolymer. Adapted with permission from Jaumaux et al."n Copyright 2020, John Wiley and Sons. Ionic soft material comprised by gelatin (22 wt $\%$ ) and $\mathrm{ChCl} /$ Ethylene glycol (1:2): (b) compressive loading-unloading curve, (c) optical images of a square knot created by tying two rectangular gel strips together (top) and the square knot being stretched by hand (bottom), and (d) optical images of the DES gel material being stretched to 300 \% strain. Adapted with permission from Panzer et al." Copyright 2019, Royal Society of Chemistry.

Alternatively, Hong et al.o observed that an ionic soft material prepared with $\mathrm{ChCl} /$ Urea/Glycerol, PAAM polymer matrix and cellulose pulp, showed stretchable behavior and superior mechanical properties in comparison to the gel obtained in water (without DES). The DES gel material was able to withstand a weight as high as $500 \mathrm{~g}$ and recovery rapidly without any cracks after the release of compressive force. These superior mechanical properties are associated with the unique structure of the network, consisting of hydrogen bonds between the DES and the PAAM chains ${ }^{30}$ Moreover, Panzer and coworkers ${ }^{36}$ reported on ionic soft materials, comprised by 
gelatin and $\mathrm{ChCl} /$ Ethylene glycol (1:2), showing highly stretchable behaviors, while their analogous hydrogels did not. The DES gel material containing $22 \mathrm{wt} \%$ gelatin was compressed up to $90 \%$ strain without any irreversible damage and recovered to its original shape immediately after releasing the pressure (Figure 5b). Two long strip-shaped gels also tolerated being tied into a square knot, which is very stretchable (Figure 5c). What is more, this DES gel material exhibited a fracture strain higher than $300 \%$ (Figure 5d). ${ }^{36}$

Ionic soft materials based on DESs have also shown interesting $\mathrm{pH}$-responsive behavior. In this case, the gel materials show different behaviors upon changes in the $\mathrm{pH}$ of the system. For instance, Mukesh and Prasad, ${ }^{35}$ demonstrated the preparation of DNA-based ionic gels, in which the formation of three different morphologies of DNA were obtained in the presence of $\mathrm{ChCl} /$ Ethylene glycol (1:2). The DNA was converted into $\mathrm{pH}$ reversible ionic gels, comprising aggregated DNA structures ( $\mathrm{pH} 2.98)$, spheroid shaped DNA microstructured assemblies, and $\mathrm{pH}$ responsive DNA micro-hydrogels ( $\mathrm{pH}$ 7.30). Such versatile DNA structures can find applications in slow and targeted gene or drug delivery applications. ${ }^{35}$

To conclude this section, DES based gels have already demonstrated similar properties (ionic conductivity, self-healing, stretchability and $\mathrm{pH}$-responsiveness) previously reported for ionic liquid gels or iongels. The main advantage shown by these ionic soft materials rely in the biobased nature, simple preparation and versatility of composition of the DESs. The main difference is that, since DESs are mixtures of compounds which can be volatile, the thermal stability or volatility of the obtained ionic soft materials are usually compromised as compared to iongels.

\subsection{Applications of Ionic Gels based on DESs}

Most of the applications of ionic soft materials based on DESs are found as solid electrolytes. These materials combine the ionic conductivity provided by the DES and the solid-like behavior. This combination facilitates the preparation of all solid electrochemical devices, circumventing the 
main drawback related to the leaching or flammability of liquid electrolytes. DES gel solid electrolytes have been mainly used in batteries and supercapacitors, while recent progress has also been accomplished in the application of these ionic soft materials to the field of (bio)electronics. Although this section is mainly focus on the recent trends of ionic soft materials based on DESs and their applications in energy and (bio)electronics, other promising applications in drug release, analytical separations, and wastewater treatment will be also discussed (Figure 6). Specific examples of DES gel materials will be detailed, but the main purpose is to provide an integrated perspective and consolidate current achievements.

\section{(b) (Bio)electronics}

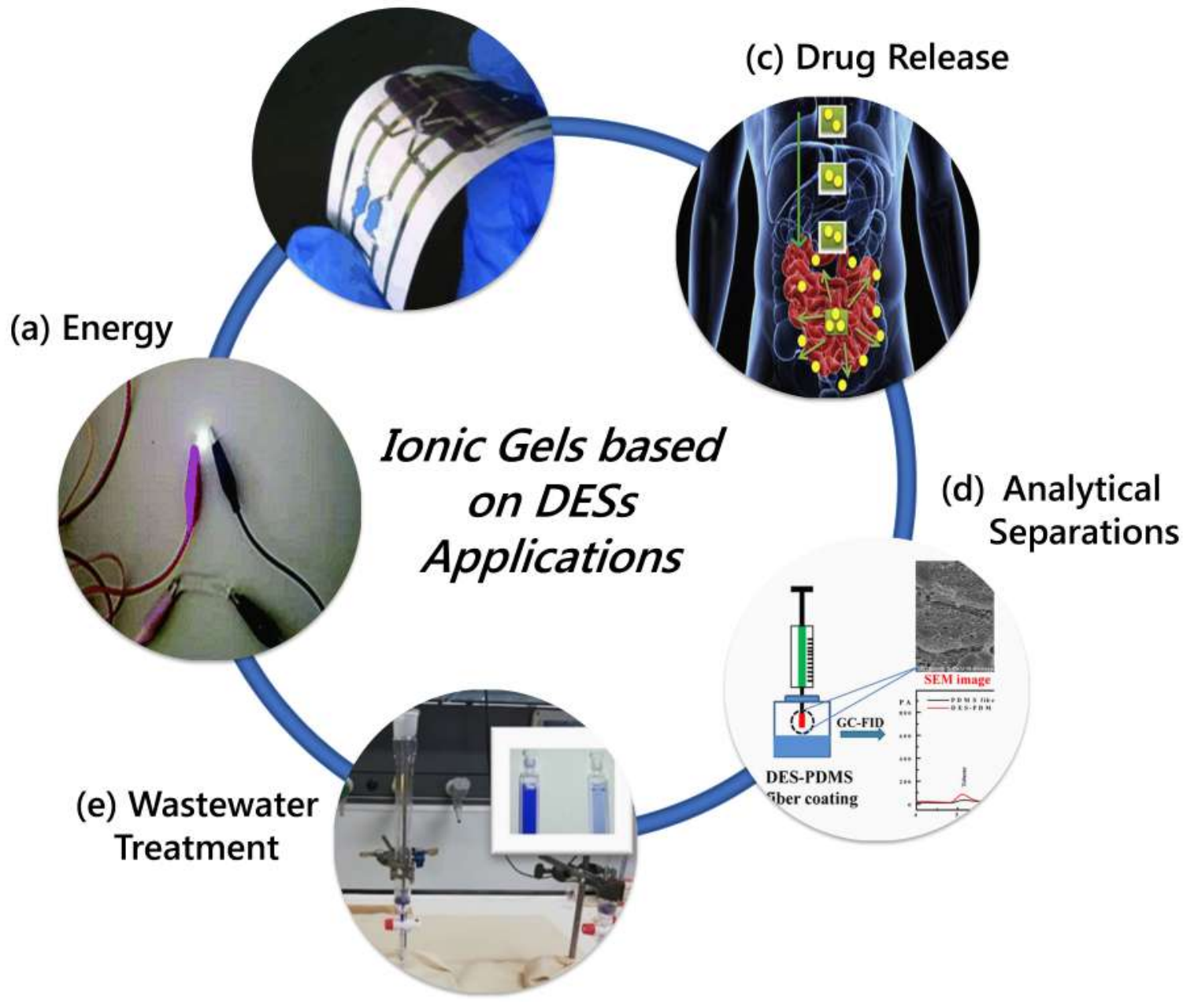


Figure 6. Perspective of applications of ionic soft materials based on DESs. (a) Energy. Adapted with permission from Hong et al. ${ }^{30}$ Copyright 2020, Royal Society of Chemistry. (b) (Bio)electronics. Adapted with permission from Pettersson et al.$^{47}$ Copyright 2015, John Wiley and Sons. (c) Drug delivery. Adapted with permission from Mokhtarpour et al..$^{29}$ Copyright 2020, Elsevier. (d) Analytical separations. Adapted with permission from $\mathrm{Li}$ et al.s Copyright 2019, Elsevier. (e) Wastewater treatment. Adapted with permission from Marullo et al ${ }^{\circledR 9}$ Copyright 2020, John Wiley and Sons.

\subsubsection{Energy}

The use of DESs as electrolytes for lithium batteries has been restricted by the low electrochemical and thermal stability of conventional DES based on organic salts, such as $\mathrm{ChCl}$ combined with urea. However, the topic is becoming popular after the recent discovery that lithium solid salts (i.e. LiTFSI) can form eutectic mixtures with a number of HBD compounds such as glymes or $\mathrm{N}$-methylacetamide. These eutectic mixtures are low viscosity liquids at room temperature showing high ionic conductivity, low flammability and wide electrochemical window, and therefore can be used as electrolytes in lithium batteries. The first example of this type of materials was reported by Joos et al. ${ }^{39}$ who reported a series of DES gel materials prepared by solgel condensation of tetraethylorthosilicate (TEOS) within LiTFSI/N-methylacetamide. These ionic soft materials, named as "eutectogels" by the authors, showed high ionic conductivity (1.46 mS $\mathrm{cm}^{-1}$ at room temperature) and wide electrochemical window $(1.1-4.7 \mathrm{~V})$. As a drawback, the thermal stability was worse than that of iongels and a weight loss after $100{ }^{\circ} \mathrm{C}$ was observed by TGA, probably due to the free $\mathrm{N}$-methylacetamide. $\mathrm{Li} / \mathrm{LiFePO}_{4}$ all solid state cells were demonstrated, showing good cyclability (100 cycles) even though a relatively low specific capacity of $105 \mathrm{mAhg}^{-1} \cdot{ }^{39}$

More recently, Joos et al. ${ }^{34}$ reported a gel polymer electrolyte of the LiTFSI/N-methylacetamide DES trapped within a polymer network formed by EGDMA/AcMo. Once again, the solid electrolytes showed some weight loss around $80{ }^{\circ} \mathrm{C}$, which was mainly attributed to evaporation of the DES. Interestingly, the prepared materials showed good flexibility, low flammability and interesting performance in $\mathrm{Li} / \mathrm{LiFePO}_{4}$ battery cells up to $130 \mathrm{mAhg}$ over 100 cycles at $\mathrm{C} / 5,^{34}$ as 
shown in Figure 7. A similar gel polymer electrolyte was also developed by Logan et al., ${ }^{32}$ who encapsulated the LiTFSI/N-methylacetamide DES within a UV-curable acrylic network based on MPEGA, HEA and PEGDA. The main advantage of this method is the fast preparation of ionic soft materials by photocuring $(<1 \mathrm{~min})$. Flexible polymer electrolytes were obtained, with ionic conductivities in the range of $0.4 \mathrm{mS} \mathrm{cm}{ }^{-1}$ and successful cycling in a $\mathrm{Li}_{4} \mathrm{Ti}_{5} \mathrm{O}_{12} / \mathrm{LiMn}_{2} \mathrm{O}_{4}$ full cells..$^{32}$

(a)

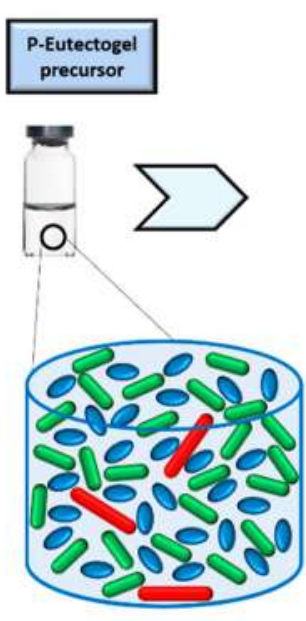

Before curing (liquid)
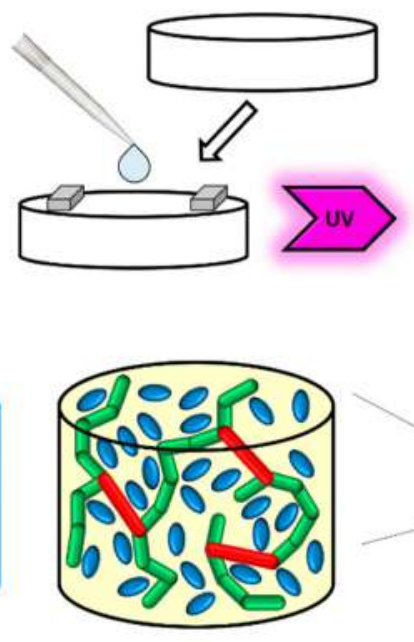

After curing (solid)

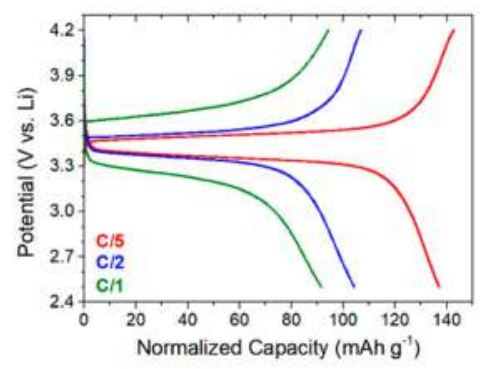

P-Eutectogel

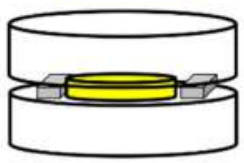

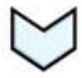
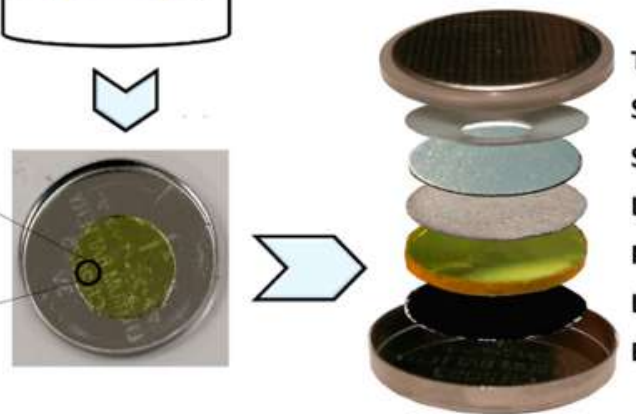

Top cap (-)

Spring

Spacer

Li anode

P-ETG

LFP@Al cathode

Bottom cap (+)
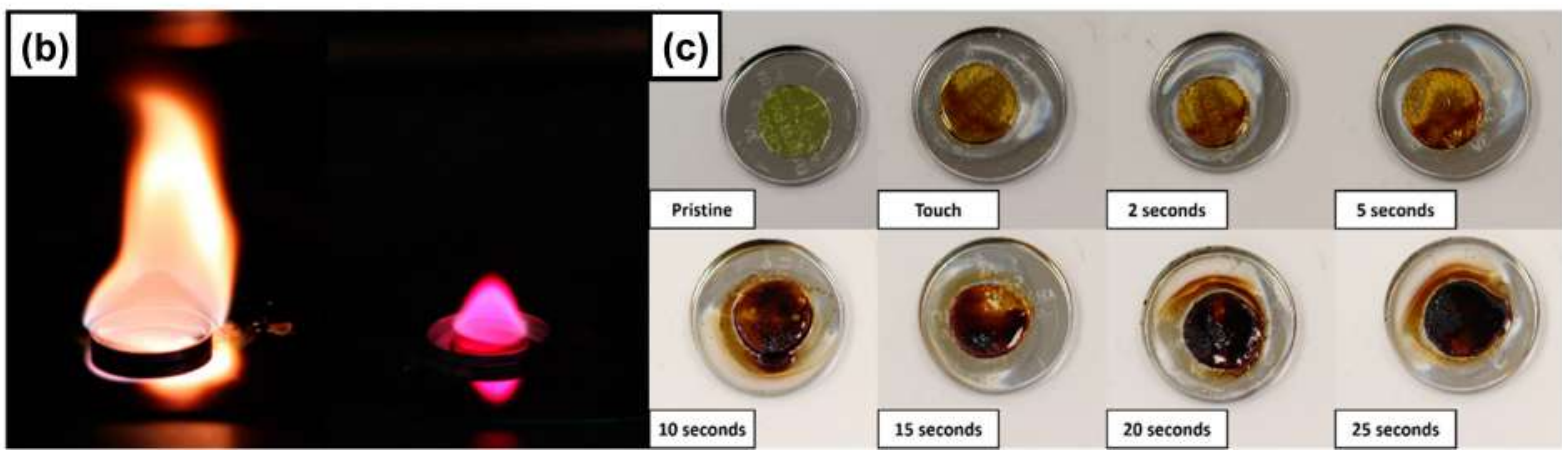

Figure 7. (a) A summary of the main steps involved in the synthesis of a DES gel polymer electrolyte, combining LiTFSI/N-methylacetamide and EGDMA/AcMO, and its further integration inside a coin cell for cycling studies. (b) Optical photograph of the ignited conventional liquid electrolyte (i.e. $200 \mu \mathrm{L}$ of $1 \mathrm{M}$ LiPF6 in EC/DEC) and the DES gel polymer electrolyte. (c) Samples of the DES gel polymer electrolyte (radius $5 \mathrm{~mm}$, height $1 \mathrm{~mm}$ ) before and after ignition and exposure to the torch for 0 (one swift touch), 2, 5 , 10, 15, 20 and 25 seconds. Adapted with permission from Joos et al..$^{34}$ Copyright 2020, American Chemical Society. 
A very recent last example was reported by Jaumaux et al., ${ }^{31}$ who further tuned the properties of this type of gel polymer electrolytes by adding an additive, i.e. fluoroethylene carbonate (FEC), to the LiTFSI/N-methylacetamide DES and trapping it using an acrylic network formed by UPyMA and PETEA monomers. This additive is known to form excellent SEI layers, allowing to plate/strip lithium for long cycles. On the other hand, UPyMa monomer contain ureopyridine groups able to form strong hydrogen bonds, which is commonly used in self-healing materials. All in all, the prepared materials performed as engineered polymer electrolytes, showing a very good combination of properties (ionic conductivity, electrochemical window, self-healing) and good cycling performance in high voltage $\mathrm{Li} / \mathrm{Li}_{4} \mathrm{Ti}_{5} \mathrm{O}_{12}$ cells. ${ }^{31}$

The requirement of electrochemical stability of the electrolyte of other energy storage devices, such as supercapacitors or $\mathrm{Zn}$ batteries, is lower than the one of lithium batteries. For this reason, more classical ChCl-based DESs have been proposed for these applications. As an illustrative example, Qin and Panzer, ${ }^{33}$ fabricated DES gel materials based on $\mathrm{ChCl} /$ Ethylene glycol and HEMA/PEGDA network. The gel polymer electrolytes showed a very high ionic conductivity of $5.7 \mathrm{mS} \mathrm{cm}-1$ at $25^{\circ} \mathrm{C}$. The flexible supercapacitor prototype with activated carbon fabric electrodes delivered a specific capacitance of $33.3 \mathrm{Fg}^{-1}$ and an energy density of $15.8 \mathrm{Wh} \mathrm{kg}^{-1.33}$ Similarly, Mukesh et al. ${ }^{27}$ reported DES gel materials of $\mathrm{ChCl} /$ Orcinol trapped within an acrylic network resulting in a highly stretchable gel. Supercapacitor cells built using thermal treated MOF as electrode and the DES gel as polyelectrolyte, delivered a maximum energy density of $33 \mathrm{WhKg}^{-1}$ and power density of $5940 \mathrm{WKg}^{-1} .^{27}$ In a very recent example, Hong et al.$^{30}$ prepared a DES gel material of $\mathrm{ChCl} / \mathrm{Urea} / \mathrm{Glycerol}$ trapped within an acrylamide network. The mechanical properties were improved by dispersion cellulose pulp into the DES. This DES gel material was applied as a solid-state electrolyte in a symmetric supercapacitor with a wide operating voltage window of $2 \mathrm{~V}$ and a high specific capacitance of $161.8 \mathrm{~F} \mathrm{g-1}$. It also exhibited a high energy density of $22.47 \mathrm{~W} \mathrm{~h}$ $\mathrm{kg}^{-1}$ and an outstanding capacity retention after more than 2000 cycles..$^{30}$ Overall, all these works 
support the notion that DES gel electrolytes are suitable for solid-state flexible supercapacitor applications.

\subsection{2. (Bio)electronics}

Electronics play an important role in our everyday life. However, creating the next generation of flexible (bio)electronic devices requires novel materials that can revolutionize this field. Highperformance, biodegradability and biocompatibility are critical issues. Tremendous efforts have been made toward developing new functional materials. ${ }^{50}$ In particular, ionic soft materials based on DESs are attracting considerable attention by virtue of their high stretchability, mechanical conformity, good transparency and bio-based nature.

With the intent of fabricate cheap, degradable, and environmentally friendly transistors, Pettersson et $a l .{ }^{47}$ used DES gel materials as the electrolytic layer in low-voltage, ion-modulated transistors (IMTs) fabricated on a paper substrate. The DESs combining $\mathrm{ChCl}$ and different hydrogen bond donors (glycerol, urea, and sorbitol) were mixed with a water-based commercially available binder and the respective solutions were spin coated to create gel-like solid materials. In particular, the IMTs built with $\mathrm{ChCl} /$ sorbitol outperformed all the other transistors tested, including the commercially available "green" reference ionic liquid (EcoEng 212) device. The IMTs with $\mathrm{ChCl} /$ sorbitol were also used to build ring oscillators, logic gates, and memories on paper. Although these were not the fast devices, they are very promising components for applications, in which the speed is not the most important factor, as for example, memory devices and sensors. $^{47}$

Ionic soft materials based on DESs have also demonstrated potential for their application in ionic skin devices. ${ }^{30,36}$ Artificial skin devices with the ability to sensitively detect stimuli, such as mechanical force and temperature changes, have been intensively investigated for applications in 
soft robotics and healthcare. ${ }^{51}$ Ionic conductive hydrogels have been long studied for this purpose, but they are susceptible to dehydration due to water evaporation. ${ }^{52}$ On the other hand, iongels endowed with negligible vapor pressure and address the volatility issue of hydrogels, but the use of ionic liquids is often challenged considering their toxicity and high production costs. In view of that, Panzer and coworkers, ${ }^{36}$ proposed highly stretchable DES gel materials based on $\mathrm{ChCl} /$ Ethylene glycol and gelatin. Several ionic skin prototype sensor devices were fabricated and promising performances were observed. Specifically, the DES gel-based strain sensor exhibited good linearity in its response and a gauge factor of $\sim 0.5$, while a pressure sensor prototype could sensitively measure pressures as low as $1 \mathrm{kPa}$. These capabilities allowed the DES gel-based ionic skin device to monitor human finger bending and multi-touch stimuli. ${ }^{36}$ Moreover, Hong et al. ${ }^{30}$ fabricated a DES gel material comprised of PAAM and $\mathrm{ChCl} / \mathrm{Urea} /$ Glycerol, possessing strainsensitive properties of conductive elastomers and exhibiting excellent real-time monitoring ability. As it can be seen from Figure 8, with the step-by-step changes in the grasping motion and tension deformation, the increase resistance of the DES gel material varied systematically. After the palm was opened, the material became completely relaxed and the resistance could be restored to its initial value..$^{30}$ In sum, the preliminary device demonstrations reinforce the idea that ionic soft materials based on DESs represent a feasible and promising non-volatile alternative to hydrogels for ionic skin applications or even for other optoelectronic devices such as electrochromics. ${ }^{40}$ 

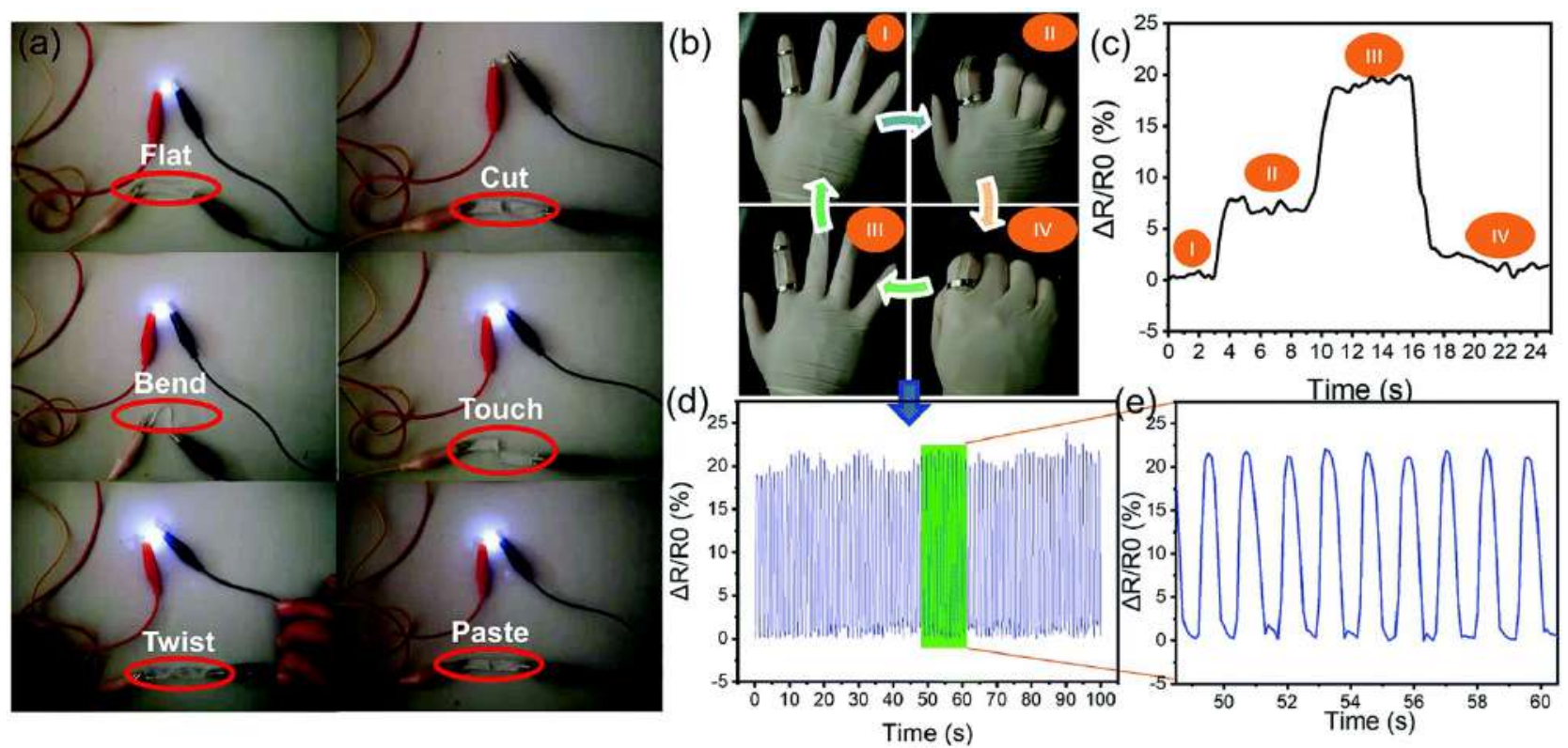

Figure 8. (a) Photographs demonstrating the electrical conductivity of an ionic soft material comprised by PAAM and $\mathrm{ChCl} / \mathrm{Urea} /$ Glycerol with various deformations. (b and $\mathrm{c}$ ) The PAAM and $\mathrm{ChCl} / \mathrm{Urea} / \mathrm{Glycerol}$ gel material as electronic skin to monitor the grasping motion and its corresponding current change. (d and e) Relative resistance of the PAAM and $\mathrm{ChCl} / \mathrm{Urea} / \mathrm{Glycerol}$ gel material as a wearable sensor in monitoring finger bending motion. Reproduced with permission from Hong et al. ${ }^{30}$ Copyright 2020, Royal Society of Chemistry

\subsubsection{Other applications of Ionic Soft Materials based on DESs}

Ionic soft materials based on DESs are finding new applications in very different technology areas. To illustrate this, in this section we will cite three examples: drug delivery, analytical separations and water purification.

Drug Release. Due to several advantages associated with DESs based on natural compounds, such as their excellent solvation properties, low toxicity and biodegradability, their corresponding ionic soft materials have been finding application in sustained drug release systems. For example, Mukesh et $a l .{ }^{28}$ reported an ionic soft material composed of HEMA and ChCl/Frutose with the ability to immobilize indomethacin, an anti-inflammatory drug. The drug-immobilized gel was 
capable to release $96 \%$ and $88 \%$ of indomethacin at $\mathrm{pH} 7.4$ and 3.8 , for $15 \mathrm{~h}$ and $20 \mathrm{~h}$, respectively, at physiological human body temperature. Importantly, this DES gel material did not inhibit the growth of mammalian cells in vitro and was found to be compatible with the human blood, thus suggesting its potential for therapeutic applications as $\mathrm{pH}$-sensitive hydrophobic drug carrier for oral application, as well as in tissue engineering. ${ }^{28}$ More recently, Mokhtarpour et al. ${ }^{29}$ developed an ionic soft system containing an anti-cancer drug (sunitinib malate), a natural and therapeutic DES (ChCl/ascorbic acid) and HEMA as polymer matrix. The gel formulation was found to be non-toxic to HN-5 cells and stable up to 6 months without any degradation. The drug release studies indicated that the release was higher at $\mathrm{pH} 1.2$ than $\mathrm{pHs} 6.8$ and 7.4. This $\mathrm{pH}$ dependent releasing behavior is of particular importance to achieve tumor-targeted drug delivery with gels (Figure 6c). ${ }^{29}$ Overall, the outcomes of both studies suggested that ionic soft materials based on DESs possess many favorable features to be used as unique drug delivery systems.

Analytical Separations. With the development of this new class of solvents, DESs have been used for different purposes in analytical chemistry. ${ }^{53}$ In particular, ionic soft materials based on DESs have shown to be good alternatives to IL-based materials in analytical methods. Yousefi et $a l .{ }^{41}$ prepared magnetic bucky gels using $\mathrm{ChCl} /$ urea and magnetic multi-walled carbon nanotube (MMWCNT) and used them in the development of dispersive solid-phase extraction (dSPE). During the injection of the gel material into the aqueous sample, the hydrophilic DES solubilized in water and increased the dispersion of MMWCNT, enlarging the contact surface between analyte and sorbent, which lead to higher extraction efficiency and shorter extraction time. In addition, the $\mathrm{ChCl}$ acted as salting out agent and enhanced the efficiency of extracting organochlorine pesticides from aqueous solutions. ${ }^{41}$ The same authors also developed a facile headspace single drop 
microextraction method (HS-SDME) for the determination of volatile aromatic hydrocarbons in water and urine samples. ${ }^{42}$ This time using a hydrophobic magnetic bucky gel formed by combining $\mathrm{ChCl} / \mathrm{Chlorophenol}$ and MMWCNT. Magnetic susceptibility, high viscosity, high sorbing ability, and tunable extractability of organic analytes made this DES bucky gel a suitable acceptor phase for HS-SDME. It was also possible to increase the droplet stability by using a magnet, overcoming the main disadvantage of common HS-SDME methods. ${ }^{42}$ Furthermore, as illustrated in Figure 6d, Li et al. ${ }^{48}$ used a hydrophobic DES $\left(\mathrm{N}_{8881} \mathrm{Cl} /\right.$ Ethylparaben) as additive of adsorptive coating (PDMS) for head space solid phase microextraction (HS-SPME). The PDMSDES fiber exhibited excellent extraction capability towards toluene, ethylbenzene and o-xylene when compared to pristine PDMS and other reported hybrid sol-gel PDMS coatings. The results revealed that the hydrophobic DES incorporated in the sol-gel solution reduced cracking and shrinking of the fiber and form porous structure on the coating surface, which was responsible for a more efficient extraction performance. ${ }^{48}$

Wastewater treatment. Current concerns for sustainability and the environment make low-impact materials desirable for wastewater treatment. With this premise in mind, Marullo et al. ${ }^{49}$ tested fully natural supramolecular gels, comprising L-amino acids and DES ( $\mathrm{ChCl} /$ Phenylacetic acid), as sorbents for the removal of dyes from wastewater. The best results were obtained with the Lphenylalanine-based gel. In terms of adsorption capacity, this gel is competitive with other gelbased sorbent systems, with a value of $1930 \mathrm{mg} / \mathrm{g}$ reached at a high concentration of rhodamine B (479 $\mathrm{mg} / \mathrm{L})$. Interestingly, this gel material was loaded onto columns to decolorize flowing solutions (Figure 6e), achieving $85 \%$ of removal efficiency in only $10 \mathrm{~min}$ and allowing its reuse for at least 4 cycles. ${ }^{49}$ Indeed, this work unveiled that ionic soft materials based on DESs have two 
main advantages perfectly fitting sustainability requirements. First, these sorbent materials present very low environmentally impact, since they are entirely made of bio-based components. Second, their recycle does not require intermediate washing, significantly decreasing the amount of waste deriving from the whole process.

\section{PERSPECTIVES AND OPPORTUNITIES}

Deep eutectic solvents are an emerging family of ionic compounds, which can expand the scope of soft-solid materials with tailor-made optical, ionic, electrical, mechanical and solvation properties. In particular, the possibility of using biobased chemicals for the DES formation opened up new exciting perspectives in the design of green materials, which are essential in modern sustainable chemistry. Moreover, considering that the physical and chemical properties of DESs can be adjusted by selecting both the nature and the ratio of the H-bonding constituents, novel DESs are designed daily and many additional new compounds continue to be discovered. Therefore, plenty of DESs, as for instance the ones based on betaines, have not yet been exploited yet in the fabrication of soft ionic materials.

Particularly, it should be noted that the majority of DESs used in the development of ionic soft materials were hygroscopic since they were composed of ionic and hydrophilic materials. In real applications, water uptake by the ionic soft material from the surrounding atmosphere is hard to avoid, and this maybe result in gel swelling and DES leaching problems. Hydrophobic DESs is an emerging subclass of DESs, which allows the use of these compounds in applications that require direct contract with water.$^{20}$ The main difference in terms of chemical structures, either ionic or neutral, lies in the presence of long alkyl chains that decrease the importance of hydrophilic domains or groups. Since 2015, when the potential of hydrophobic DESs was revealed by Kroon's group, s4 several hydrophobic DESs have been prepared from hydrophobic salts and other hydrophobic organic natural compounds ${ }^{20}$ However, there is only two reported examples of DESbased gel materials, which have been prepared using hydrophobic DESs, namely 
$\mathrm{ChCl} /$ Chlorophenol and $\mathrm{N}_{8881} \mathrm{Cl} /$ Ethylparaben. ${ }^{42,}{ }^{48}$ Thus, we consider that there is an open research opportunity in the use of hydrophobic DESs for the preparation of water-stable ionic soft materials.

One more important point arising from the composition of the ionic soft materials is related with the gelator component. Different types of gelators, such as linear polymers, polymer networks, biopolymers, supramolecular compounds or sol-gel derived silica networks, were used. Nevertheless, the use of block copolymers has not yet been reported and the sol-gel derived silica networks are under-exploited. It is well known, from previous experiences with hydrogels, organogels and iongels, that ABA type triblock copolymers are good gelators if A is a block based on a polymer non-soluble in the solvent, while B is a block of a soluble polymer. Another possibility within the synthetic polymer family could be the use of the polymeric versions of DESs (Figure 3), ${ }^{11,26,55}$ which have not been explored so far as gelators to form three-dimensional networks with improved compatibility within the different components.

From the results reported in the last 5 years, it clearly appears that ionic soft materials based on DESs exhibit key properties (ionic conductivity, mechanical and rheological properties) and additional material functionalities (self-healing, stretchability and $\mathrm{pH}$-responsivenes), which are close to those of traditional ionic liquid based iongels. Additionally, like iongels, the physicochemical properties of these emerging ionic soft materials can be tuned by changing the nature of both the DES and the gelator, making possible the preparation of task-specific materials for diverse applications. However, in terms of fabrication and when compared to ionic liquids, DESs have remarkable advantages, stemming from (1) their lower price since DESs can be prepared from readily accessible chemicals, (2) their convenient synthesis (100\% atom economy), and (3) their low toxicity, especially those DESs derived from natural renewable resources. Hence, we consider that there is plenty of room for the development of unique soft ionic materials based on DESs offering additional properties or functionalities, such as shape memory or hyperelasticity, thermo- or photo-responsiveness, mixed ionic/electronic properties, (photo)luminescent, magnetic, bioactivity, among others. Following this line of research, interesting applications will be easily 
envisaged, especially in those fields where the preparation of high-tech and low cost materials is critical.

Finally, these emerging ionic soft materials based on DESs have been shown to be alternatives to iongels in the fields of energy, (bio)electronics, drug delivery, analytical chemistry, and wastewater treatment. Contrary to iongels, which use as $\mathrm{CO}_{2}$ separation membranes has attracted much attention, DES-based gel materials have not been proposed yet for gas separation applications. This can be associated to the hydrophilic nature of the DESs used so far, or even due to the possible evaporation of the DES components from the gel matrix at specific pressure and temperature conditions. Nonetheless, DESs have recently been tested as liquid phases in supported liquid membranes and composite membranes, showing great potential for different gas separation

applications. ${ }^{56-59}$ In view of that, we believe that the field of gas separation membrane technology is still open for ionic soft materials based on DESs.

All in all, more studies on the building principles and properties will be needed in the near future in order to gain more insights about these emerging soft ionic materials and to find new applications.

\section{AUTHOR INFORMATION}

\section{Corresponding Authors}

*Liliana C. Tomé (lilianasofi.carvalho@ehu.eus); *David Mecerreyes (david.mecerreyes@ehu.es)

\section{Author Contributions}

The manuscript was written through contributions of all authors. All authors have given approval to the final version of the manuscript.

\section{Notes}


The authors declare no competing financial interest.

\section{Biographies}

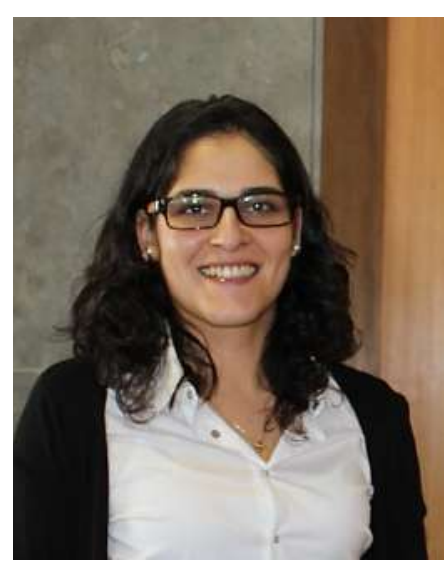

Liliana C. Tomé was born in 1985 in Alcácer do Sal, Portugal. She obtained her master's degree in Materials Derived from Renewable Resources from University of Aveiro, Portugal (2008). In 2014, she completed her PhD in Engineering and Technology Sciences - Chemical Engineering by Instituto de Tecnologia Química e Biológica António Xavier (ITQB NOVA), Portugal. During 2015-2018, she was a post-doctoral researcher at ITQB NOVA. Currently, she is a Marie Sklodowska-Curie Individual Fellowship at POLYMAT, University of the Basque Country, Spain. Her main areas of research are centered on the development of iongels and poly(ionic liquid)based materials for gas separation and bioelectronics.

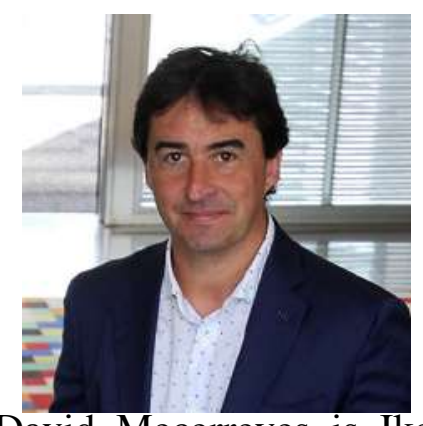

David Mecerreyes is Ikerbasque Research Professor at POLYMAT-University of the Basque Country (Spain). He received his PhD from University of Liege in 1998. After postdoc (19982000) at IBM Almaden Research Center (California), he moved to Spain to work at CIDETEC. In 2010, he moved to POLYMAT where he became the leader of the Innovative Polymers Group. 
His area of expertise is polymer synthesis of ionic and electroactive polymers such as poly(ionic liquid)s, polymer electrolytes, redox polymers and PEDOT type conductive polymers. Current research interests involve the application of innovative polymers in emerging technologies such as organic batteries and bioelectronic devices. His research has been supported by the European Research Council, Basque Government and Spanish Ministry of Science and Education.

\section{ACKNOWLEDGMENTS}

Liliana C. Tomé has received funding from the European Union's Horizon 2020 research and innovation programme under the Marie Sklodowska-Curie grant agreement no. 745734 . This work was supported by Marie Sklodowska-Curie Research and Innovation Staff Exchanges (RISE) project under the grant agreement No 823989 "IONBIKE".

\begin{tabular}{|c|c|}
\hline \multicolumn{2}{|c|}{ ABBREVIATIONS } \\
\hline AcMo & acryloylmorpholine \\
\hline CNT & carbon nanotubes \\
\hline $\mathrm{ChCl}$ & choline chloride \\
\hline DBS & 1,3:2,4-Dibenzylidene-d-sorbitol \\
\hline $\operatorname{DES}(s)$ & deep eutectic solvent(s) \\
\hline DNA & deoxyribonucleic acid \\
\hline $\mathrm{dSPE}$ & dispersive solid-phase extraction \\
\hline EGDMA & ethylene glycol dimethacrylate \\
\hline FEC & fluoroethylene carbonate \\
\hline $\operatorname{HBA}(s)$ & hydrgen bond acceptor(s) \\
\hline $\operatorname{HBD}(s)$ & hydrogen bond donor(s) \\
\hline HEA & 2-hydroxyethyl acrylate \\
\hline HEMA & 2-hydroxyethyl methacrylate \\
\hline HS-SDME & headspace single drop microextraction method \\
\hline HS-SPME & head space solid phase microextraction \\
\hline IMT & ion-modulated transistors \\
\hline
\end{tabular}


LiTFSI lithium bis(trifluoromethanesulfonyl)imide

LMWG low-molecular weight gelator

MMWCNT magnetic multi-walled carbon nanotube

MPEGA poly(ethylene glycol) methyl ether acrylate

NIPAM N-isopropylacrylamide

PAAM polyacrylamide

PETEA pentaerythritol tetraacrylate

PEGDA poly(ethylene glycol) diacrylate

PDMS polydimethylsiloxane

TEOS tetraethylorthosilicate

UPyMA 2-(3-(6-methyl-4-oxo-1,4-dihydropyrimidin-2-yl)ureido) ethyl methacrylate

VA vinyl acetate

\section{REFERENCES}

1. Fuller, J., Ionic Liquid-Polymer Gel Electrolytes. J. Electrochem. Soc. 1997, 144, L67.

2. Noda, A.; Watanabe, M., Highly Conductive Polymer Electrolytes Prepared by in Situ Polymerization of Vinyl Monomers in Room Temperature Molten Salts. Electrochim. Acta 2000, $45,1265-1270$.

3. Kimizuka, N.; Nakashima, T., Spontaneous Self-Assembly of Glycolipid Bilayer Membranes in Sugar-Philic Ionic Liquids and Formation of Ionogels. Langmuir 2001, 17, 6759-6761.

4. Forsyth, M.; Porcarelli, L.; Wang, X.; Goujon, N.; Mecerreyes, D., Innovative Electrolytes Based on Ionic Liquids and Polymers for Next-Generation Solid-State Batteries. Acc. Chem. Res. 2019, 52, 686-694.

5. Isik, M.; Lonjaret, T.; Sardon, H.; Marcilla, R.; Herve, T.; Malliaras, G. G.; Ismailova, E.; Mecerreyes, D., Cholinium-Based Ion Gels as Solid Electrolytes for Long-Term Cutaneous Electrophysiology. J. Mater. Chem. C 2015, 3, 8942-8948.

6. Tomé, L. C.; Marrucho, I. M., Ionic Liquid-Based Materials: A Platform to Design Engineered Co2 Separation Membranes. Chem. Soc. Rev. 2016, 45, 2785-2824. 
7. Smith, E. L.; Abbott, A. P.; Ryder, K. S., Deep Eutectic Solvents (Dess) and Their Applications. Chem. Rev. 2014, 114, 11060-11082.

8. Abbott, A. P.; Boothby, D.; Capper, G.; Davies, D. L.; Rasheed, R. K., Deep Eutectic Solvents Formed between Choline Chloride and Carboxylic Acids: Versatile Alternatives to Ionic Liquids. J. Am. Chem. Soc. 2004, 126, 9142-9147.

9. Florindo, C.; Lima, F.; Ribeiro, B. D.; Marrucho, I. M., Deep Eutectic Solvents: Overcoming 21st Century Challenges. Curr. Opin. Green Sustain. Chem. 2019, 18, 31-36.

10. Carriazo, D.; Serrano, M. C.; Gutiérrez, M. C.; Ferrer, M. L.; del Monte, F., Deep-Eutectic Solvents Playing Multiple Roles in the Synthesis of Polymers and Related Materials. Chem. Soc. Rev. 2012, 41, 4996-5014.

11. Mota-Morales, J. D.; Sánchez-Leija, R. J.; Carranza, A.; Pojman, J. A.; del Monte, F.; LunaBárcenas, G., Free-Radical Polymerizations of and in Deep Eutectic Solvents: Green Synthesis of Functional Materials. Prog. Polym. Sci. 2018, 78, 139-153.

12. Roda, A.; Matias, A. A.; Paiva, A.; Duarte, A. R. C., Polymer Science and Engineering Using Deep Eutectic Solvents. Polymers 2019, 11, 912.

13. Wagle, D. V.; Zhao, H.; Baker, G. A., Deep Eutectic Solvents: Sustainable Media for Nanoscale and Functional Materials. Acc. Chem. Res. 2014, 47, 2299-2308.

14. Abbott, A. P.; Capper, G.; Davies, D. L.; Rasheed, R. K.; Tambyrajah, V., Novel Solvent Properties of Choline Chloride/Urea Mixtures. Chem. Commun. 2003, 70-71.

15. Paiva, A.; Craveiro, R.; Aroso, I.; Martins, M.; Reis, R. L.; Duarte, A. R. C., Natural Deep Eutectic Solvents - Solvents for the 21st Century. ACS Sustain. Chem. Eng. 2014, 2, 1063-1071.

16. Francisco, M.; van den Bruinhorst, A.; Kroon, M. C., Low-Transition-Temperature Mixtures (Lttms): A New Generation of Designer Solvents. Angew. Chem. Int. Ed. 2013, 52, 3074-3085.

17. Anastas, P.; Eghbali, N., Green Chemistry: Principles and Practice. Chem. Soc. Rev. 2010, 39, 301-312.

18. Tomé, L. I. N.; Baião, V.; da Silva, W.; Brett, C. M. A., Deep Eutectic Solvents for the Production and Application of New Materials. Appl. Mater. Today 2018, 10, 30-50.

19. Liu, Y.; Friesen, J. B.; McAlpine, J. B.; Lankin, D. C.; Chen, S.-N.; Pauli, G. F., Natural Deep Eutectic Solvents: Properties, Applications, and Perspectives. J. Nat. Prod. 2018, 81, 679-690.

20. Florindo, C.; Branco, L. C.; Marrucho, I. M., Quest for Green-Solvent Design: From Hydrophilic to Hydrophobic (Deep) Eutectic Solvents. ChemSusChem 2019, 12, 1549-1559. 
21. Rodriguez Rodriguez, N.; van den Bruinhorst, A.; Kollau, L. J. B. M.; Kroon, M. C.; Binnemans, K., Degradation of Deep-Eutectic Solvents Based on Choline Chloride and Carboxylic Acids. ACS Sustain. Chem. Eng. 2019, 7, 11521-11528.

22. Mota-Morales, J. D.; Gutiérrez, M. C.; Sanchez, I. C.; Luna-Bárcenas, G.; del Monte, F., Frontal Polymerizations Carried out in Deep-Eutectic Mixtures Providing Both the Monomers and the Polymerization Medium. Chem. Commun. 2011, 47, 5328-5330.

23. Sánchez-Leija, R. J.; Pojman, J. A.; Luna-Bárcenas, G.; Mota-Morales, J. D., Controlled Release of Lidocaine Hydrochloride from Polymerized Drug-Based Deep-Eutectic Solvents. $J$. Mater. Chem. B 2014, 2, 7495-7501.

24. Mota-Morales, J. D.; Gutiérrez, M. C.; Ferrer, M. L.; Jiménez, R.; Santiago, P.; Sanchez, I. C.; Terrones, M.; Del Monte, F.; Luna-Bárcenas, G., Synthesis of Macroporous Poly(Acrylic Acid)Carbon Nanotube Composites by Frontal Polymerization in Deep-Eutectic Solvents. J. Mater. Chem. A 2013, 1, 3970-3976.

25. Isik, M.; Ruiperez, F.; Sardon, H.; Gonzalez, A.; Zulfiqar, S.; Mecerreyes, D., Innovative Poly(Ionic Liquid)S by the Polymerization of Deep Eutectic Monomers. Macromol. Rapid Commun. 2016, 37, 1135-1142.

26. Isik, M.; Zulfiqar, S.; Edhaim, F.; Ruiperez, F.; Rothenberger, A.; Mecerreyes, D., Sustainable Poly(Ionic Liquids) for Co2 Capture Based on Deep Eutectic Monomers. ACS Sustain. Chem. Eng. 2016, 4, 7200-7208.

27. Mukesh, C.; Gupta, R.; Srivastava, D. N.; Nataraj, S. K.; Prasad, K., Preparation of a Natural Deep Eutectic Solvent Mediated Self Polymerized Highly Flexible Transparent Gel Having Super Capacitive Behaviour. RSC Adv. 2016, 6, 28586-28592.

28. Mukesh, C.; Upadhyay, K. K.; Devkar, R. V.; Chudasama, N. A.; Raol, G. G.; Prasad, K., Preparation of a Noncytotoxic Hemocompatible Ion Gel by Self-Polymerization of Hema in a Green Deep Eutectic Solvent. Macromol. Chem. Phys. 2016, 217, 1899-1906.

29. Mokhtarpour, M.; Shekaari, H.; Shayanfar, A., Design and Characterization of Ascorbic Acid Based Therapeutic Deep Eutectic Solvent as a New Ion-Gel for Delivery of Sunitinib Malate. $J$. Drug. Deliv. Sci. Technol. 2020, 56, 101512.

30. Hong, S.; Yuan, Y.; Liu, C.; Chen, W.; Chen, L.; Lian, H.; Liimatainen, H., A Stretchable and Compressible Ion Gel Based on a Deep Eutectic Solvent Applied as a Strain Sensor and Electrolyte for Supercapacitors. J. Mater. Chem. C 2020, 8, 550-560. 
31. Jaumaux, P.; Liu, Q.; Zhou, D.; Xu, X.; Wang, T.; Wang, Y.; Kang, F.; Li, B.; Wang, G., Deep-Eutectic-Solvent-Based Self-Healing Polymer Electrolyte for Safe and Long-Life LithiumMetal Batteries. Angew. Chem. Int. Ed. 2020, 59, 9134-9142.

32. Logan, M. W.; Langevin, S.; Tan, B.; Freeman, A. W.; Hoffman, C.; Trigg, D. B.; Gerasopoulos, K., Uv-Cured Eutectic Gel Polymer Electrolytes for Safe and Robust Li-Ion Batteries. J. Mater. Chem. A 2020, 8, 8485-8495.

33. Qin, H.; Panzer, M. J., Chemically Cross-Linked Poly(2-Hydroxyethyl Methacrylate)Supported Deep Eutectic Solvent Gel Electrolytes for Eco-Friendly Supercapacitors. Chemelectrochem 2017, 4, 2556-2562.

34. Joos, B.; Volders, J.; da Cruz, R. R.; Baeten, E.; Safari, M.; Van Bael, M. K.; Hardy, A. T., Polymeric Backbone Eutectogels as a New Generation of Hybrid Solid-State Electrolytes. Chem. Mater. 2020, 32, 3783-379.

35. Mukesh, C.; Prasad, K., Formation of Multiple Structural Formats of DNA in a Bio-Deep Eutectic Solvent. Macromol. Chem. Phys. 2015, 216, 1061-1066.

36. Qin, H.; Owyeung, R. E.; Sonkusale, S. R.; Panzer, M. J., Highly Stretchable and Nonvolatile Gelatin-Supported Deep Eutectic Solvent Gel Electrolyte-Based Ionic Skins for Strain and Pressure Sensing. J. Mater. Chem. C 2019, 7, 601-608.

37. Depoorter, J.; Mourlevat, A.; Sudre, G.; Morfin, I.; Prasad, K.; Serghei, A.; Bernard, J.; Fleury, E.; Charlot, A., Fully Biosourced Materials from Combination of Choline Chloride-Based Deep Eutectic Solvents and Guar Gum. ACS Sustain. Chem. Eng. 2019, 7, 16747-16756.

38. Vioux, A.; Viau, L.; Volland, S.; Le Bideau, J., Use of Ionic Liquids in Sol-Gel; Ionogels and Applications. C.R. Chim. 2010, 13, 242-255.

39. Joos, B.; Vranken, T.; Marchal, W.; Safari, M.; Van Bael, M. K.; Hardy, A. T., Eutectogels: A New Class of Solid Composite Electrolytes for Li/Li-Ion Batteries. Chem. Mater. 2018, 30, 655662.

40. Wang, R.; Zhang, S.; Su, Y.; Liu, J.; Ying, Y.; Wang, F.; Cao, X., Semi-Solid State Electrochromic Device Based on Deep Eutectic Solvent Gel Electrolyte and Transparent $\mathrm{Au}$ Modified Fto Electrode. Electrochim. Acta 2017, 258, 328-335.

41. Yousefi, S. M.; Shemirani, F.; Ghorbanian, S. A., Deep Eutectic Solvent Magnetic Bucky Gels in Developing Dispersive Solid Phase Extraction: Application for Ultra Trace Analysis of 
Organochlorine Pesticides by Gc-Micro Ecd Using a Large-Volume Injection Technique. Talanta 2017, 168, 73-81.

42. Yousefi, S. M.; Shemirani, F.; Ghorbanian, S. A., Enhanced Headspace Single Drop Microextraction Method Using Deep Eutectic Solvent Based Magnetic Bucky Gels: Application to the Determination of Volatile Aromatic Hydrocarbons in Water and Urine Samples. J. Sep. Sci. 2018, 41, 966-974.

43. Chen, S.; Zhang, B.; Zhang, N.; Ge, F.; Zhang, B.; Wang, X.; Song, J., Development of SelfHealing D-Gluconic Acetal-Based Supramolecular Ionogels for Potential Use as Smart Quasisolid Electrochemical Materials. ACS Appl. Mater. Inter. 2018, 10, 5871-5879.

44. Marullo, S.; Meli, A.; Giannici, F.; D’Anna, F., Supramolecular Eutecto Gels: Fully Natural Soft Materials. ACS Sustain. Chem. Eng. 2018, 6, 12598-12602.

45. Ruiz-Olles, J.; Slavik, P.; Whitelaw, N. K.; Smith, D. K., Self-Assembled Gels Formed in Deep Eutectic Solvents: Supramolecular Eutectogels with High Ionic Conductivity. Angew. Chem. Int. Ed. 2019, 58, 4173-4178.

46. Delbecq, F.; Delfosse, P.; Laboureix, G.; Paré, C.; Kawai, T., Study of a Gelated Deep Eutectic Solvent Metal Salt Solution as Template for the Production of Size-Controlled Small Noble Metal Nanoparticles. Colloids Surf. A Physicochem. Eng. Asp. 2019, 567, 55-62.

47. Pettersson, F.; Remonen, T.; Adekanye, D.; Zhang, Y.; Wilén, C.-E.; Österbacka, R., Environmentally Friendly Transistors and Circuits on Paper. ChemPhysChem 2015, 16, 12861294.

48. Li, T.; Song, Y.; Xu, J.; Fan, J., A Hydrophobic Deep Eutectic Solvent Mediated Sol-Gel Coating of Solid Phase Microextraction Fiber for Determination of Toluene, Ethylbenzene and OXylene in Water Coupled with Gc-Fid. Talanta 2019, 195, 298-305.

49. Marullo, S.; Meli, A.; Dintcheva, N. T.; Infurna, G.; Rizzo, C.; D'Anna, F., Environmentally Friendly Eutectogels Comprising L-Amino Acids and Deep Eutectic Solvents: Efficient Materials for Wastewater Treatment. ChemPlusChem 2020, 85, 301-311.

50. Huang, S.; Liu, Y.; Zhao, Y.; Ren, Z.; Guo, C. F., Flexible Electronics: Stretchable Electrodes and Their Future. Adv. Funct. Mater. 2019, 29, 1805924.

51. Hammock, M. L.; Chortos, A.; Tee, B. C.-K.; Tok, J. B.-H.; Bao, Z., 25th Anniversary Article: The Evolution of Electronic Skin (E-Skin): A Brief History, Design Considerations, and Recent Progress. Adv. Mater. 2013, 25, 5997-6038. 
52. Wang, H.; Wang, Z.; Yang, J.; Xu, C.; Zhang, Q.; Peng, Z., Ionic Gels and Their Applications in Stretchable Electronics. Macromol. Rapid Commun. 2018, 39, 1800246.

53. Shishov, A.; Bulatov, A.; Locatelli, M.; Carradori, S.; Andruch, V., Application of Deep Eutectic Solvents in Analytical Chemistry. A Review. Microchem. J. 2017, 135, 33-38.

54. van Osch, D. J. G. P.; Zubeir, L. F.; van den Bruinhorst, A.; Rocha, M. A. A.; Kroon, M. C., Hydrophobic Deep Eutectic Solvents as Water-Immiscible Extractants. Green Chem. 2015, 17, 4518-4521.

55. Ren'ai, L.; Zhang, K.; Chen, G.; Su, B.; Tian, J.; He, M.; Lu, F., Green Polymerizable Deep Eutectic Solvent (Pdes) Type Conductive Paper for Origami 3d Circuits. Chem. Commun. 2018, 54, 2304-2307.

56. Jiang, B.; Dou, H.; Wang, B.; Sun, Y.; Huang, Z.; Bi, H.; Zhang, L.; Yang, H., Silver-Based Deep Eutectic Solvents as Separation Media: Supported Liquid Membranes for Facilitated Olefin Transport. ACS Sustain. Chem. Eng. 2017, 5, 6873-6882.

57. Dou, H.; Jiang, B.; Zhang, L.; Xu, M.; Sun, Y., Synergy of High Permeability, Selectivity and Good Stability Properties of Silver-Decorated Deep Eutectic Solvent Based Facilitated Transport Membranes for Efficient Ethylene/Ethane Separation. J. Membr. Sci. 2018, 567, 39-48.

58. Karimi, M. B.; Mohammadi, F.; Hooshyari, K., Non-Humidified Fuel Cells Using a Deep Eutectic Solvent (Des) as the Electrolyte within a Polymer Electrolyte Membrane (Pem): The Effect of Water and Counterions. Phys. Chem. Chem. Phys. 2020, 22, 2917-2929.

59. Lin, H.; Gong, K.; Ying, W.; Chen, D.; Zhang, J.; Yan, Y.; Peng, X., Co2-Philic Separation Membrane: Deep Eutectic Solvent Filled Graphene Oxide Nanoslits. Small 2019, 15, 1904145. 


\section{TOC Graphic}

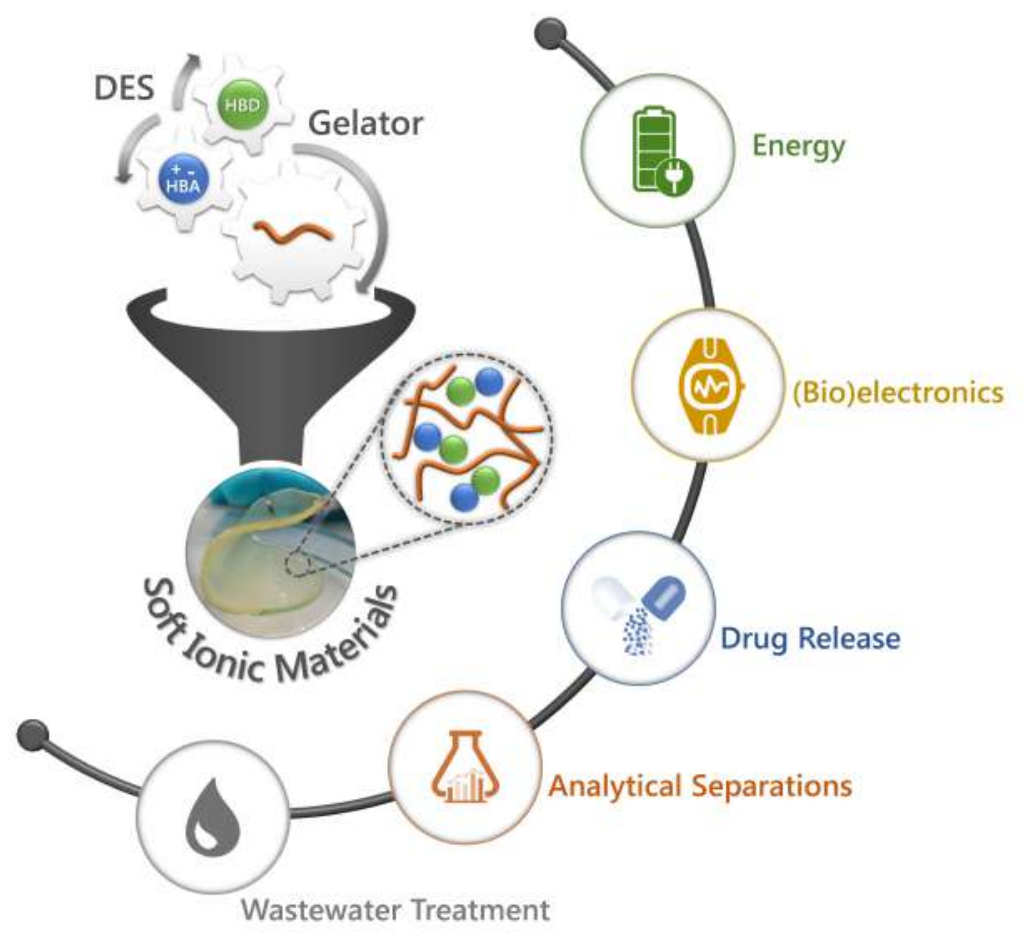

